\title{
SOLUÇÕES PERIÓDICAS \\ DE UMA EQUAÇÃO DIFERENCIAL NO PLANO \\ COM RETARDAMENTO E CONTINUAÇÃO GLOBAL
}

Margarete Teresa Zanon Baptistini

Tese apresentada ao Instituto de Ciências Matemáticas de São Carlos, da Universidade de São Paulo, para obtenção do grau de Doutor em Ciências (Matemática).

Orientador: Prof. Dr. Plácido Zoega Táboas

São Carlos

1990 
Às minhas filhas

Flávia e Mariana 


\section{Agradecimentos}

Ao Prof. Dr. Plácido Z. Táboas, pela demonsuusuo de amizade e confiança, pelo exemplo de seriedade profissional e pela dedicaçào e entusiasmo com que conduziu este trabalho, o meu muito obrigada.

Aos colegas do Departamento de Matemática da UFSCar e do ICMSC-USP, pelo apoio nos momentos dificeis e pelo incentivo recebido, tão importante nesta fase da minha vida profissional.

Às minhas amigas Alice e Yolanda, companheiras na UFSCar que, por utilização de recursos computacionais, não só permitiram que este tivesse tal forma de apresentação, mas o fizeram como se fosse em causa própria.

Finalmente, agradeço à minha familia, por ter procurado compreender os meus muitos momentos de ausência, em especial ao Pedrinho, que sempre soube valorizar o meu trabalho. 


\section{Abstract}

The existence of periodic solutions of the one-parametric family of planar differential-delay equations

$$
\dot{x}(t)=-\alpha x(t)+\alpha F(x(t-1))
$$

with $\alpha \in \mathbf{R}$, is studied.

A sequence of Hopf bifurcation is determined under fairly general hypotheses on $F$. We also state a global continuation result. 


\section{Índice}

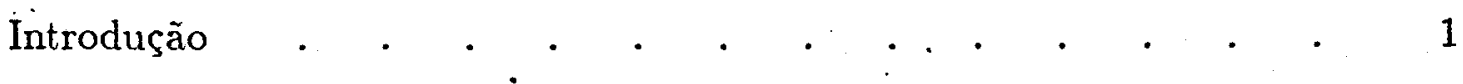

capítulo 1

Os autovalores da equação linearizada

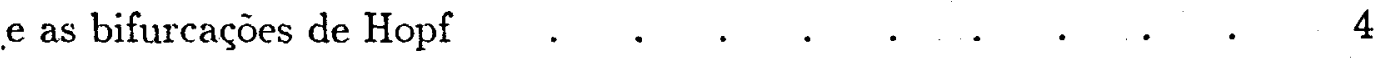

capítulo 2

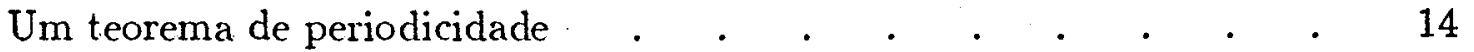

capítulo 3

Estimativas exponenciais uniformes . . . . . . . . . $\quad$. 35

capitulo 4

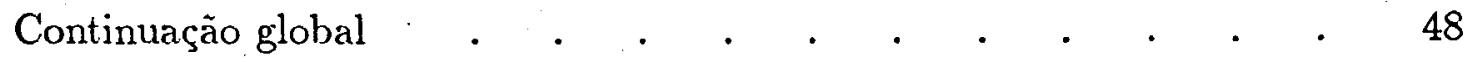

Referências . . . . . . . . . . . . . . 66 


\section{Introdução}

O objetivo principal deste trabalho é contribuir para a descrição da dinâmica determinada pela família de equações diferenciais com retardamento

$$
\dot{x}(t)=-\alpha x(t)+\alpha F(x(t-1)) .
$$

onde $x \doteq \operatorname{col}\left(x_{1}, x_{2}\right) \in \mathbf{R}^{2}, F: \mathbf{R}^{2} \longrightarrow \mathbf{R}^{2}$ de classe $\mathcal{C}^{2}$, e $\alpha$ um parâmetro real.

Tal equação tem sido exaustivamente estudada no caso escalar, isto é, quando $x \in$ $\mathbf{R}$ e $F$ é uma função real, e as aplicações têm ocorrido com grande ênfase nas ciências biológicas. Contribuições na área são dadas, por exemplo, em [4]-[12], [14] e, em [11], Mallet-Paret dá uma descrição global da dinâmica de (1), mais especificamente êle obtem uma decomposição de Morse de um atrator compacto maximal.

Resultados de estudos da equação (1) no plano, ao que nos consta, apareceram só recentemente. Nesta direção, há o trabalho de P.Z. Táboas, [16], em que é estudada uma equação semelhante à (1) e no qual foram inspirados muitos dos resultados aqui contidos.

Um problema importante-que se coloca é o de determinar soluções globais de (1), (definidas em $(-\infty,+\infty)$ ), já que, usualmente, elas consistem de conjuntos $\alpha$-limite ou $\omega$-limite de outras soluções e desempenham, portanto, papel fundamental no estudo do comportamento assintótico. Esses fatos justificam a ênfase deste trabalho no estudo de soluções periódicas.

No capítulo 1 é feito um estudo da equação característica e prova-se, para uma certa classe de funções $F$, a existência de uma infinidade de valores positivos e negativos do parâmetro $\alpha$ onde ocorrem bifurcações de Hopf.

O capítulo 2 é uma aplicação de um teorema de ponto fixo através do qual obtém-se existência de soluções periódicas da equação (1) com período $T>4$, para $\alpha$ maior que um 
certo valor $\alpha_{0}>0$.

Os resultados do capítulo 3 constituem uma extensão dos resultados de Hale em $[\mathbf{9}, 1.5]$ para uma família a um parâmetro de equações diferenciais diferença, com o parâmetro $\alpha$ variando em certos subconjuntos compactos da reta real.

O capítulo 4 trata da continuação global de uma bifurcação de Hopf, isto é, motivados pelos resultados obtidos nos capítulos 1 e 2, provamos a existência de um ramo contínuo ilimitado de soluções periódicas de (1) emanante de $\alpha_{0}$, sob condições adicionais à função $F$.

A escolha, para o nosso estudo, de $\alpha$ variando no semi-eixo real positivo é motivada, em parte, pela equivalência de (1) à equação com retardamento $\alpha$ :

$$
\dot{y}(t)=-y(t)+F(y(t-\alpha))
$$

através de uma mudança na escala do tempo. Além disso, o nosso resultado do capítulo 2 sugere estudar um ramo de soluções periódicas com período $>4$, ao passo que para $a<0$ as bifurcações de Hopf dão origem a soluções con período $<4$.

Em todo este trabalho denotaremos por $\mathcal{C}=\mathcal{C}\left([-1,0], \mathbf{R}^{2}\right)$ o espaço de Banach definido por

$$
\mathcal{C}\left([-1,0], \mathbf{R}^{2}\right)=\left\{\varphi:[-1,0] \rightarrow \mathbf{R}^{2} \mid \varphi \text { é contínua }\right\}
$$

munido da norma

$$
\|\varphi\|=\sup _{-1 \leq \theta \leq 0}|\varphi(\theta)|
$$

onde $|\cdot|$ denota a norma usual do $\mathbf{R}^{2}$.

Dados $t_{0} \geq 0, A \geq 0$ e uma função $x \in \mathcal{C}\left(\left[t_{0}-1, t_{0}+A\right], \mathbf{R}^{2}\right)$, para cada $t \in\left[t_{0}, t_{0}+A\right]$, denotaremos por $x_{t} \in \mathcal{C}$ a função definida por

$$
\text { - } x_{t}(\theta)=x(t+\theta), \quad-1 \leq \theta \leq 0 \text {. }
$$

Se $\phi \in \mathcal{C}$, uma solução $x(t)$ de (1) que coincide com $\phi$ em $[-1,0]$ deve satisfazer

$$
\begin{aligned}
& x(t)=\phi(t), \quad t \in[-1,0] \\
& x(t)=\epsilon^{-\alpha t} \phi(0)+\alpha \int_{0}^{t} \epsilon^{-\alpha(t-s)} F(x(s-1)) d s, t \geq 0
\end{aligned}
$$


e reciprocamente. A partir desta expressão e pelo processo recursivo em intervalos de comprimento um mostra-se existência e unicidade da solução en $[-1, \infty)$. Para naiores informações sobre a teoria geral dessas equações, veja [9]. 


\section{Capítulo 1}

\section{Os Autovalores da Equação Linearizada e as Bifurcações de Hopf}

Neste capítulo, considerando a família a um parâmetro de equações diferenciais autônomas não lineares da forma

$$
\dot{x}(t)=-\alpha x(t)+\alpha F(x(t-1)) .
$$

onde $x=\operatorname{col}\left(x_{1}, x_{2}\right) \in \mathbf{R}^{2}, F: \mathbf{R}^{2} \longrightarrow \mathbf{R}^{2}$ de classe $\mathcal{C}^{2}, F(0)=0, F^{\prime}(0) \neq 0$ e $\alpha$ um parâmetro real, é nosso propósito estabelecer existência de soluções periódicas para certos valores do parâmetro $\alpha$, as quais emanam da solução nula - as chamadas Bifurcações de Hopf. É feito um estudo mais amplo da localização dos autovalores da parte linear de $(1.1-\alpha)$, que será útil em outros capítulos; tratamentos gerais são dados em [1] e [15]. Daremos maior ênfase a valores positivos de $\alpha$, objetivando resultados futuros.

As equações $(1.1-\alpha)$ com $F$ munida da hipótese seguinte:

$$
x_{2} F_{1}(x)>0, \text { se } x_{2} \neq 0 \text { e } x_{1} F_{2}(x)<0, \text { se } x_{1} \neq 0
$$

têm linearizaçöes próximas à origem dadas por:

$$
\dot{x}(t)=-\alpha x(t)+\alpha B x(t-1)
$$

onde

$$
B:=\left(\begin{array}{cc}
0 & \delta_{1} \\
\delta_{2} & 0
\end{array}\right), \quad \delta_{1}:=\frac{\partial F_{1}}{\partial x_{2}}(0) \geq 0, \quad \delta_{2}:=\frac{\partial F_{2}}{\partial x_{1}}(0) \leq 0
$$


Assumiremos em todo este capítulo $\delta=\sqrt{-\delta_{1} \delta_{2}}>0$, a menos que se diga algo em contrário.

Uma condição necessária e suficiente para a existência de uma solução não trivial de (1.2- $\alpha)$ da forma $x(t)=e^{\lambda t} u, u \in \mathbf{R}^{2}$, é que $\lambda$ seja raiz da chamada equação característica de $(1.2-\alpha)$ :

$$
\operatorname{det} H_{\alpha}(\lambda)=0, \quad H_{\alpha}(\lambda):=(\lambda+\alpha) I-\alpha B e^{-\lambda}
$$

onde $I$ representa a matriz identidade de ordem 2; ou equivalentemente

$$
(\lambda+\alpha)^{2} e^{2 \lambda}=-\delta^{2} \alpha^{2} .
$$

Observemos que $\lambda=0$ é a única raiz real e ocorre somente quando $\alpha=0$.

Além disso, $\lambda=\lambda(\alpha)$ é raiz de $(1.4-\alpha)$ se e somente se, seu complexo conjugado $\bar{\lambda}$ também o for ; podemos, então, restringir nosso estudo ao semiplano superior $\Im \lambda>0$.

Lema 1.1. Não existe raiz de $(1.4-\alpha)$ sobre as retas $3 \lambda=\left(k+\frac{1}{2}\right) \pi, k=0,1,2, \ldots$

Demonstração: Se $\lambda=a+b i,(1.4-\alpha)$ é equivalente ao sistema

$$
\left\{\begin{array}{cl}
e^{2 a}\left\{\left[(a+\alpha)^{2}-b^{2}\right] \cos 2 b-2 b(a+\alpha) \operatorname{sen} 2 b\right\} & =-\delta^{2} \alpha^{2} \\
{\left[(a+\alpha)^{2}-b^{2}\right] \operatorname{sen} 2 b+2 b(a+\alpha) \cos 2 b} & =0 .
\end{array}\right.
$$

que se reduz, para $b=\left(k+\frac{1}{2}\right) \pi, k=0,1,2, \ldots$, a

o qual é incompatível. $\diamond$

$$
\left\{\begin{array}{cl}
\dot{e}^{2 a}\left[(a+\alpha)^{2}-b^{2}\right] & =\delta^{2} \alpha^{2} \\
2 b(a+\alpha) & =0
\end{array}\right.
$$

Segue do Lema 1.1 que toda raiz característica $\lambda \operatorname{com} \Im \lambda>0$ deve pertencer a alguma das faixas:

$$
\begin{gathered}
S_{0}:=\left\{\lambda \in \mathbf{C} \mid 0<\Im \lambda<\frac{\pi}{2}\right\} \\
S_{k}:=\left\{\lambda \in \dot{C} \mid\left(k-\frac{1}{2}\right) \pi<\Im \lambda<\left(k+\frac{1}{2}\right) \pi\right\}, k=1,2, \ldots
\end{gathered}
$$


Lema 1.2. Em cada faixa $S_{k}, k \geq 0$, a equação $(1.4-\alpha)$ é equivalente a

$$
(\lambda+\alpha) \epsilon^{\lambda}=(-1)^{k} i \delta|\alpha|
$$

Demonstração:

$$
(\lambda+\alpha)^{2} e^{2 \lambda}=-\delta^{2} \alpha^{2} \Longleftrightarrow(\lambda+\alpha) \epsilon^{\lambda}= \pm i \delta|\alpha|
$$

Tomando $\lambda=a+b i$ :

$$
\begin{cases}(a+\alpha) \cos b-b \operatorname{sen} b & =0 \\ (a+\alpha) \operatorname{sen} b+b \cos b & = \pm \delta|\alpha| \epsilon^{-a}\end{cases}
$$

Segue da primeira equação de (1.6) que $b(a+\alpha) \operatorname{sen} b \cos b=b^{2} \operatorname{sen}^{2} b \geq 0$, isto é, $(a+\alpha) \operatorname{sen} b$ e $b \cos b$ têm sempre o mesmo sinal.

$O$ lema fica demonstrado observando que, em $S_{k}$, o sinal de $b \cos b$ é $(-1)^{k} . \diamond$

Lema 1.3. Para $\delta>1$ e $\alpha>0$, fica determinada de modo único uma sequência $\left(\lambda_{k}\right)$ de raizes caracteristicas tal que $\lambda_{k}=b_{k} i \in S_{k}$, sen $b_{k}=(-1)^{k} 1 / \delta$, e os valores $\alpha_{k}$ correspondentes são dados por

$$
\alpha_{k}=\frac{b_{k}}{\sqrt{\delta^{2}-1}}, k=0,1,2 \ldots
$$

\section{Demonstração:}

$$
\lambda=b i \text { em }(\lambda+\alpha) e^{\lambda}=(-1)^{k} i \delta \alpha \text { implica que }
$$

$$
\left\{\begin{array}{cl}
\alpha & =b \operatorname{tg} b \\
\alpha \operatorname{sen} b+b \cos b & =(-1)^{k} \delta \alpha
\end{array}\right.
$$

e então

$$
\operatorname{sen} b=(-1)^{k} \frac{1}{\delta} \quad e \quad \cos b=(-1)^{k} \frac{\sqrt{\delta^{2}-1}}{\delta}
$$

para todo inteiro $k \geq 0$, de onde segue o resultado. $\diamond$

$$
\alpha_{0}=\frac{b_{0}}{\sqrt{\delta^{2}-1}}, \quad 0<b_{0}=\operatorname{arcsen} \frac{1}{\delta}<\frac{\pi}{2}
$$


o qual corresponde à raiz característica $\lambda_{0}=b_{0} i$ em $S_{0}$ é um valor do parâmetro que tem um papel importante em todo este trabalho, como logo sèrá percebido.

A sequência $\left(\alpha_{k}\right)$ dada acima é tal que $0<\alpha_{0}<\alpha_{1}<\ldots \rightarrow+\infty$ quando $k \rightarrow+\infty$, já que $b_{k}=b_{0}+k \pi$ para $k \geq 1$.

Lema 1.4. Se $\delta>0$, cada faixa $S_{k}, k \geq 0$, contém precisamente uma raiz de $(1.4-a)$ para cada $\alpha \neq 0$, e essa raiz é simples.

Demonstração: para $\alpha>0, \lambda=a+b i \in S_{k}, k \geq 0$, em $(1.5-\alpha)$ implica que

$$
\left\{\begin{array}{cl}
a+\alpha & =b \operatorname{tg} b \\
(a+\alpha) \operatorname{sen} b+b \cos b & =(-1)^{k} \delta \alpha \epsilon^{-a}
\end{array}\right.
$$

e então,

$$
\delta \alpha e^{\alpha}=(-1)^{k} \frac{b e^{b \operatorname{tg} b}}{\cos b}
$$

Definindo $\beta=\delta \alpha \epsilon^{\alpha}$, segue que

$$
\frac{d \beta}{d \alpha}=\delta e^{\alpha}(1+\alpha)>0
$$

para $\alpha>0$ e $\beta((0, \infty))=(0, \infty)$.

Por outro lado,

$$
\begin{gathered}
\beta=\Lambda(b)=(-1)^{k} \frac{b e^{b \operatorname{tg} b}}{\cos b} \\
\frac{d \beta}{d b}=(-1)^{k} e^{b \operatorname{tg} b} \sec b\left[(1+b \operatorname{tg} b)^{2}+b^{2}\right]>0
\end{gathered}
$$

pois sinal de $\sec b=(-1)^{k}$ em $S_{k}, k \geq 0$.

Além disso $\Lambda(b) \rightarrow 0$ quando $b \rightarrow 0^{+}$ou $b \rightarrow\left(k-\frac{1}{2}\right) \pi^{+}$e $\Lambda(b) \rightarrow+\infty$ quando $b \rightarrow\left(k-\frac{1}{2}\right) \pi^{-}$, para $k=1,2, \ldots$

- Assim, em cada faixa $S_{k}, b=\Im \lambda$ é uma função estritamente crescente de $\alpha>0$, com domínio $(0, \infty)$ e imagem $\left(0, \frac{\pi}{2}\right)$ em $S_{0}$ e $\left(\left(k-\frac{1}{2}\right) \pi,\left(k+\frac{1}{2}\right) \pi\right)$ em $S_{k}, k \geq 1$. 
De $(1.8-\alpha)$ segue que, em cada faixa $S_{k}, a=\Re \lambda$ é também uma função de $a$, para $\alpha \in(0, \infty)$ e

$$
\frac{d a}{d \alpha}=\frac{a(1+a+\alpha)+b^{2}}{\alpha\left[(1+a+\alpha)^{2}+b^{2}\right]}
$$

Para $\alpha<0,(1.4-\alpha)$ é equivalente a

$$
\left\{\begin{array}{cl}
a+\alpha & =b \operatorname{tg} b \\
(a+\alpha) \operatorname{sen} b+b \cos b & =(-1)^{k+1} \delta \alpha e^{-a}, k \geq 0
\end{array}\right.
$$

e então

$$
\widetilde{\beta}=\delta \alpha e^{\alpha}=(-1)^{k+1} \frac{b e^{b \operatorname{tg} b}}{\cos b}
$$

Em vista disso, podemos afirmar que, em cada faixa $S_{k}, b=\Im \lambda$ é uma função estritamente crescente de $\alpha$ para $\alpha<-1$ e estritamente decrescente se $-1<\alpha<0$.

$a=\Re \lambda$, função de $\alpha$ para $\alpha<0$, é tal que

$$
\frac{d a}{d \alpha}=-1-\frac{(\alpha+1)\left(a+\alpha+(a+\alpha)^{2}+b^{2}\right)}{\alpha\left[(1+a+\alpha)^{2}+b^{2}\right]} .
$$

Observando que $\Im \lambda \neq 0$ para $\alpha \neq 0$, a parte final do lema segue de

$$
\frac{d}{d \lambda}\left[(\lambda+\alpha) e^{\lambda}-(-1)^{k} \delta|\alpha| i\right]=e^{\lambda}(1+\lambda+\alpha)
$$

Lema 1.5. Se $\delta>1$ e $\alpha>0$, em cada faixa $S_{k}$, as raizes $\lambda=\lambda(\alpha)$ da equação $(1.4-\alpha)$, são tais que $\Re \lambda<\ln \delta$ e $\lim _{\alpha \rightarrow+\infty} \lambda=\ln \delta+i\left(k+\frac{1}{2}\right) \pi, k=0,1,2, \ldots$

Demonstração: $\operatorname{De}(1.8-\alpha)$ segue que

$$
\left\{\begin{array}{cl}
a+\alpha & =b \operatorname{tg} b \\
e^{a} b \sec b & =(-1)^{k} \delta \alpha
\end{array}\right.
$$

e então $e^{a}\left(1+\frac{a}{\alpha}\right)=(-1)^{k} \delta \operatorname{sen} b$.

- Pelo lema 1.4, $\lim _{\alpha \rightarrow+\infty}(\dot{-}-1)^{k} \delta \operatorname{sen} b=\delta$.

Para $\alpha$ suficientemente grande, $a>0$, e então 


$$
e^{a}\left(1+\frac{a}{\alpha}\right)>e^{a}
$$

Assim $a=a(\alpha)$ fica limitada quando $\alpha \rightarrow+\infty \mathrm{e}$

$$
\delta=\lim _{\alpha \rightarrow+\infty} \epsilon^{a}\left(1+\frac{a}{\alpha}\right)=\lim _{\alpha \rightarrow+\infty} e^{a}
$$

isto é, $a=\Re \lambda \rightarrow \ln \delta$ quando $\alpha \rightarrow+\infty$.

As demais afirmações seguem da demonstraçãodo Lema 1.4. $\diamond$

Lema 1.6. Se $\lambda_{k}=a_{k}+b_{k} i \in S_{k}, k=0,1,2 \ldots$ são raizes de $(1.4-\alpha)$ para algum $\alpha>0$, então $a_{0}>a_{1}>\ldots \rightarrow-\infty$.

Demonstração: A equação $(1.4-\alpha)$, através da mudança de variável $\lambda+\alpha=u$ e da reparametrização dada pelo difeomorfismo $\beta=\alpha \delta \epsilon^{\alpha}$ é equivalente a

$$
u^{2} e^{2 u}=-\beta^{2}
$$

É claro que a cada solução $\lambda=\bar{a}+\bar{b} i$ da equação $(1.4-\alpha)$ para algum $\alpha>0$ corresponde biunivocamente a solução $u=(\bar{a}+\alpha)+\bar{b} i$ da equação (1.11) para $\beta=\alpha \delta \epsilon^{\alpha}$.

Segue então do Lema 1.4 que para cada $\beta>0$, cada faixa $S_{k}, k \geq 0$, contém uma única raiz de (1.11) e tal raiz é simples.

O lema estará demonstrado se a ordenação das partes reais valer para as raizes de (1.11).

Se $u^{2} e^{2 u}=-\beta^{2}$ e $u=a+b i$, então

$$
\left(a^{2}+b^{2}\right) e^{2 a}=\beta^{2}
$$

Para $\beta=\pi$ a equação. (1.12) define $b$ como função de $a, a \in(-\infty, 0]$ e tal função é decresćente, já que

$$
\frac{d b}{d a}=\frac{-1}{b}\left[\pi^{2} \epsilon^{-2 a}+a\right]<0, \text { para } a \leq 0
$$

Assim $a_{0}>a_{1}=0>\ldots>a_{k}>\ldots, \operatorname{com} a_{k}+b_{k} i=u_{k} \in S_{k}$, para $k \geq 0$. 
Suponhamos por um momento que tal ordenação seja falsa para algum parâmetro $\beta>0$.

Como $\Re u$ é uma função contínua de $\beta$ em cada faixa $S_{k}$, existirá um parâmetro $\bar{\beta}$ para o qual $a_{k}=a_{l}=a \operatorname{com} k>l$.

Mas então, pela equação (1.12):

$$
\left(a^{2}+b_{k}^{2}\right) e^{2 a}=\left(a^{2}+b_{l}^{2}\right) e^{2 a}
$$

o que implica que $b_{k}=b_{l}$, uma contradição. $\diamond$

Lema 1.7. Se $\alpha>0 e-1<\delta<1$, então toda solução de $(1.4-\alpha)$ satisfaz $\Re \lambda<0$.

Demonstração:

$$
(\lambda+\alpha)^{2} e^{2 \lambda}=-\delta^{2} \alpha^{2} \Longrightarrow|\lambda+\alpha|=|\delta| \alpha \epsilon^{-\Re \lambda}
$$

Se $\Re \lambda \geq 0$, então $\alpha \leq|\lambda+\alpha| \leq \alpha e^{-\Re \lambda} \leq \alpha$ e assim $|\lambda+\alpha|=\alpha$, de onde segue que $\lambda=0$, o que é absurdo para $\alpha>0$ e $-1<\delta<1$. $\diamond$

Os lemas 1.1 a 1.6 nos dão informações importantes sôbre o comportamento das raízes características no plano complexo, quando $\delta>1$ e $\alpha>0$.

Observemos que se $a=\Re \lambda \geq 0$ então $b=\Im \lambda$ é uma função estritamente crescente de $a$ em cada faixa $S_{k}$, já que, por (1.9), $\frac{d a}{d \alpha}>0$.

Para $0 \leq \Im \lambda<\frac{\pi}{2}$, a origem 0 e $\lambda_{0}=b_{0} i$ definido em (1.7) são raízes características e então $a<0$ para $\alpha \in\left(0, \alpha_{0}\right)$. Além disso $\frac{d a}{d \alpha}=0$ para no máximo um valor positivo de $b \mathrm{e}$

$$
\frac{d b}{d a}=\frac{(1+\alpha) b}{a(1+a+\alpha)+b^{2}}
$$

para toda raiz característica $\lambda=a+b i$ tal que $\frac{d a}{d \alpha} \neq 0$.

Quando $\delta>1$ e o parâmetro $\alpha$, partindo da origem, caminha no semi-eixo positivo, ao assumir o valor $\alpha_{0}$, um par de raizes conjugadas $\lambda_{0}=\lambda\left(\alpha_{0}\right)$ e $\bar{\lambda}_{0}$ cruza transversalmente o eixo imaginário, da esquerda para a direita, pela primeira vez. A partir daí aparecem duas raízes complexas conjugadas com parte real positiva, até o parâmetro $\alpha$ assumir um 
novo valor, $\alpha_{1}$, em que um novo par, $\lambda_{1}=\lambda\left(\alpha_{1}\right)$ e $\bar{\lambda}_{1}$ cruza o eixo imaginário; assim sucessivamente. A transversalidade é garantida por $\Re \cdot \lambda^{\prime}\left(\dot{\alpha}_{k}\right)>0$ para $k=0,1 ; 2, \ldots$

Na figura (1.1) tomamos $\delta=\sqrt{2}$. Ela foi obtida em computador e mostra o lugar geométrico das raízes características $\lambda=a+b i$ nas diversas faixas. Quando $\alpha$ varia desde 0 até $+\infty$, a parte real de $\lambda$ varia desde $-\infty$ até $\ln \sqrt{2}$, sobre as curvas indicadas, obedecendo à ordenação estabelecida no lema 1.6 .

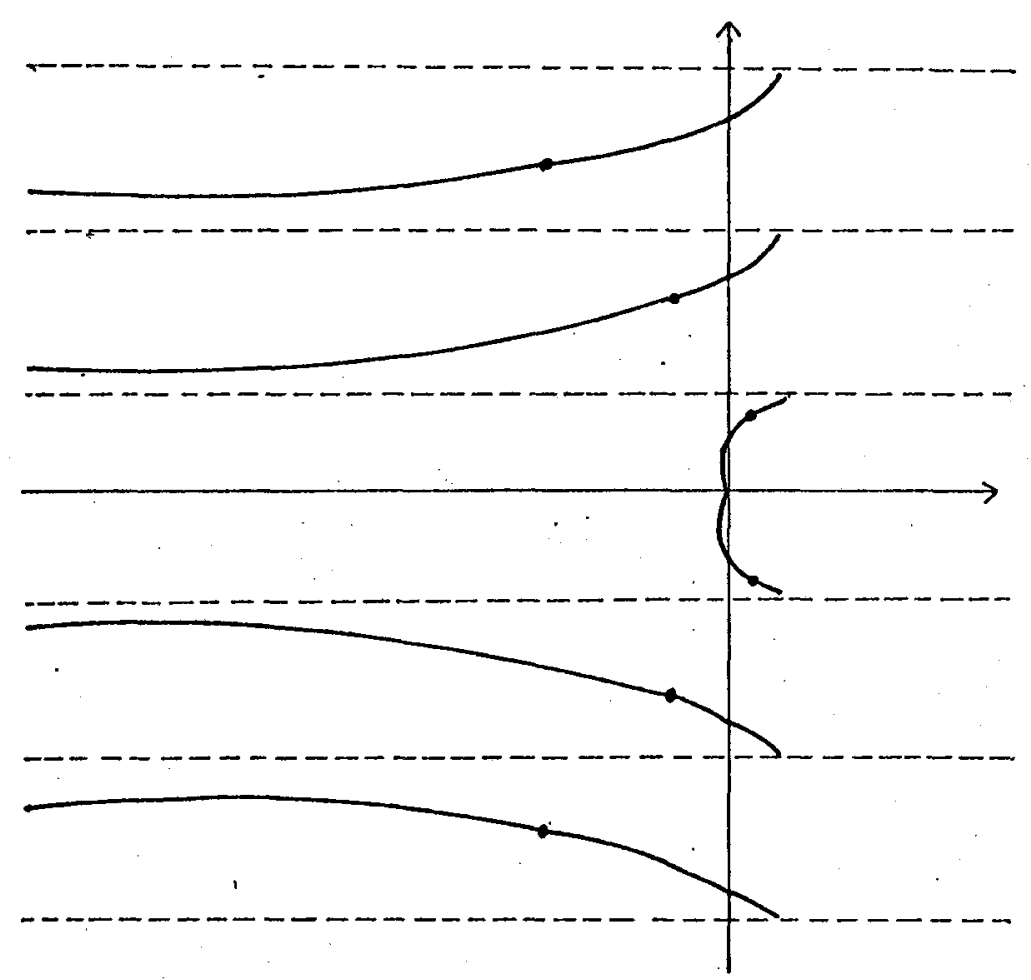

figura 1.1

Esses resultados e o Teorema da Bifurcação de Hopf em [9] conduzem-nos ao:

Teorema 1.1. Suponhamos $\delta>1$. Então existe uma sequência $0<\alpha_{0}<\alpha_{1}<\ldots \rightarrow+\infty$ de valores do parâmetro $\alpha$ para os quais a equação $(1.1-\alpha)$ tem uma bifurcação de Hopf local em $\alpha=\alpha_{k}, k=0,1,2, \ldots$ O ramo que emana de $\alpha_{k}$ representa soluções $\omega$-periódicas, $\operatorname{com} \omega$ próximo de $\frac{2 \pi}{b_{k}}, b_{k}$ definido univocamente por

$$
b_{k}=\operatorname{arcsen}(-1)^{k} \frac{1}{\delta} \in\left(\left(k-\frac{1}{2}\right) \pi,\left(k+\frac{1}{2}\right) \pi\right) \cap(0, \infty), k=0,1,2, \ldots
$$

Observemos que o caso $\alpha<0$ foi considerado nos Lemas 1.1, 1.2 e 1.4. 
O próximo lema é o análogo ao Lema 1.3 para $\alpha<0$.

Lema 1.8. Para $\delta>1$ e $\alpha<0$, fica determinada de modo único uma sequência $\left(\lambda_{k}\right)$ de raizes caracteristicas tal que $\lambda_{k}=c_{k} i \in S_{k}$, sen $c_{k}=(-1)^{k+1} 1 / \delta$, e os valores $a_{k}$ correspondentes são

$$
\alpha_{k}=\frac{-c_{k}}{\sqrt{\delta^{2}-1}}, k=1,2, \ldots
$$

Demonstração: Análoga à do Lema 1.3, tomando agora a equação

$$
(\lambda+\alpha) e^{\lambda}=(-1)^{k+1} i \delta \alpha
$$

Observemos que se $\lambda \in S_{0}$ é raiz de $(1.4-\alpha) \operatorname{com} \alpha<0$, então $\Re \lambda \neq 0$. Mais ainda, da equação $a+\alpha=b \operatorname{tg} b$, segue que $\Re \lambda>-\alpha \cdot$ para $\lambda \in S_{0}$.

No lema $1.8, c_{k}=c_{1}+(k-1) \pi$ para $k \geq 2 \operatorname{com} c_{1} \in\left(\frac{\pi}{2}, \pi\right), c_{1}=\operatorname{arcsen}(1 / \delta)$ e a correspondente sequência $\left(\alpha_{k}\right) \rightarrow-\infty$ quando $k \rightarrow+\infty$.

Além disso, por (1.10),

$$
\Re \lambda^{\prime}\left(\alpha_{k}\right)=-1-\frac{\left(\alpha_{k}+1\right)\left(\alpha_{k}+\alpha_{k}^{2}+c_{k}^{2}\right)}{\alpha_{k}\left[\left(1+\alpha_{k}\right)^{2}+c_{k}^{2}\right]} \neq 0
$$

se, e somente se,

$$
c_{k}^{2} \neq \frac{-2 \alpha_{k}\left(1+\alpha_{k}\right)^{2}}{1+2 \alpha_{k}}, k=1,2, \ldots
$$

e uma condição suficiente para isso ocorrer é que $\delta<\sqrt{\pi^{2}+1}$.

Eștamos em condições de enunciar um teorema análogo ao Teorema 1.1, para o caso $\alpha<0$.

Teorema 1.2. Seja $1<\delta^{\circ}<\sqrt{\pi^{2}+1}$. Existe uma sequência $0>\alpha_{0}>\alpha_{1}>\ldots \rightarrow-\infty$, de valores do parâmetro $\alpha$, para os quais a equação $(1.1-\alpha)$ tem uma bifurcação de Hopf 
1 Os autovalores da equaçäo linearizada e as bifurcaçōes de Hopf

local em $\alpha=\alpha_{k}, k=1,2 \ldots O$ ramo que emana de $\alpha_{k}$ representa soluções $\omega$-periódicas com $\omega$ próximo de $\frac{2 \pi}{c_{k}}, c_{k}$ definido univocamente por

$$
c_{k}=\operatorname{arcsen}(-1)^{k+1} \frac{1}{\delta} \in\left(\left(k-\frac{1}{2}\right) \pi,\left(k+\frac{1}{2}\right) \pi\right), k=1,2, \ldots
$$




\section{Capítulo 2}

\section{Um Teorema de Periodicidade}

Os fatos centrais deste capítulo são motivados por uma idéia atribuida a Jones[10], que por sua vez inspira-se no método de Poincaré. Basicamente o que se faz é provar a existência de soluções periódicas não triviais mostrando que um certo operador de retorno definido pelo fluxo num cone do espaço de fase, tem ponto fixo não trivial. Aqui, o cone desempenha papel análogo à seção transversal do método de Poincaré.

A dificuldade desse procedimento é que a origem é um ponto fixo conhecido "a priori", o qual corresponde à solução periódica trivial. Ela é contornada pelo uso de um teorema - de ponto fixo que depende do conceito de ejetividade e garante a existência de pontos fixos não nulos.

Consideremos a família de equações diferenciais autônomas

$$
\dot{x}(t)=-\alpha x(t)+\alpha F(x(t-1))
$$

onde $x=\operatorname{col}\left(x_{1}, x_{2}\right), \alpha$ parâmetro real positivo e $F: \mathbf{R}^{2} \longrightarrow \mathbf{R}^{2}$ de classe $\mathcal{C}^{2}, F=$ $\operatorname{col}\left(F_{1}, F_{2}\right)$.

Chamemos de $x(\cdot ; \alpha, \varphi)$ a solução de $(2.1-\alpha)$ definida em $[-1, \infty)$, a qual coicide com $\varphi$ em $[-1,0], \varphi \in \mathcal{C}=\mathcal{C}\left([-1,0], \mathbf{R}^{2}\right)$.

Muitas vezes faremos uso das equações $(2.1-\alpha)$ na seguinte forma

$$
\begin{aligned}
& \dot{x}_{1}(t)=-\alpha x_{1}(t)+\alpha F_{1}(x(t-1)) \\
& \dot{x}_{2}(t)=-\alpha x_{2}(t)+\alpha F_{2}(x(t-1))
\end{aligned}
$$


As hipóteses a seguir serão sempre assumidas:

$$
x_{2} F_{1}(x)>0, \text { se } x_{2} \neq 0, \quad x_{1} F_{2}(x)<0, \text { se } x_{1} \neq 0 \quad e \quad \delta=\sqrt{-\delta_{1} \delta_{2}}>1
$$

onde

$$
\delta_{1}:=\frac{\partial F_{1}}{\partial x_{2}}(0), \quad \delta_{2}:=\frac{\partial F_{2}}{\partial x_{1}}(0)
$$

$$
\text { F é limitada :- seja } M>0 \text { tal que }|F(x)| \leq M, \forall x \in \mathbf{R}^{2} \text {. }
$$

Definindo $f(x)=F(x)-B x$, onde $B=F^{\prime}(0), \quad(2.1-\alpha)$ fica equivalente a

$$
\begin{gathered}
\dot{x}(t)=-\alpha x(t)+\alpha B x(t-1)+\alpha f(x(t-1)) \\
B:=\left(\begin{array}{cc}
0 & \delta_{1} \\
\delta_{2} & 0
\end{array}\right), \quad \delta_{1}>0, \quad \delta_{2}<0, \quad \delta=\sqrt{-\delta_{1} \delta_{2}}>1, \quad f(0)=0, \quad f^{\prime}(0)=0,
\end{gathered}
$$

$f$ contínua e $\alpha>0$.

São de importância decisiva neste e no capítulo 4 os subconjuntos fechados e convexos $\operatorname{de} \mathcal{C}$

$$
\begin{gathered}
K=\left\{\varphi \in \mathcal{C} \mid \dot{\varphi}_{1}(-1)=0, \varphi_{1}, \varphi_{2} \geq 0\right\} \\
K_{\alpha}=\left\{\varphi \in \Pi \mid e^{\alpha \theta} \varphi,(\theta) \text { monótona crescente em }[-1,0], j=1,2\right\}
\end{gathered}
$$

$\operatorname{com} \alpha>0$. Embora isto não represente qualquer vantagem adicional, observamos que tais conjuntos satisfazem às condições da definição de cone: subconjunto fechado e convexo $\mathrm{D}$ de um espaço de Banach $\mathrm{X}, \lambda D \subset D$ se $\lambda \geq 0$ e $D \cap(-D)=\{0\}$.

Dado $L>0$, denotaremos por $B_{L}$ e $D_{L}$ a bola aberta em $\mathbf{R}^{2}$ e em $\mathcal{C}$, respectivamente, isto é,

$$
\begin{aligned}
& B_{L}=\left\{x \in \mathbf{R}^{2}|| x \mid<L\right\} \\
& D_{L}=\{\varphi \in \mathcal{C} \mid\|\varphi\|<L\}
\end{aligned}
$$


e por $\bar{B}_{L}$ e $\bar{D}_{L}$ seus respectivos fechos.

Teorema 2.1. Suponhamos válidas as hipóteses (H.1) e (H.2) e seja $\alpha_{0}$ como em (1.7). Então para todo $\alpha>\alpha_{0},(2.1-\alpha)$ tem solução periódica não trivial $x(\cdot ; \alpha, \varphi), \varphi \in I_{\alpha}$, com periodo $T>4$.

A prova deste teorema segue da existência de um ponto fixo não trivial de um operador $A_{\alpha}: K_{\alpha} \rightarrow K_{\alpha}, A_{\alpha}(\varphi)=x_{\tau}(\cdot ; \alpha, \varphi)$ para algum $\tau>0$, e é consequência do Lema 2.1 a seguir, devido a Nussbaum[14], .o qual é colocado aqui numa formulação menos geral, embora suficiente para nossos propósitos. O conceito de ejetividade é de acordo com Browder [3].

Definição 2.1 : Sejam $X$ um espaço topológico, $x_{0}$ um elemento de $X, U$ uma vizinhança aberta de $x_{0}$ em $X$ e $A: U-\left\{x_{0}\right\} \rightarrow X$, uma função contínua. O ponto $x_{0}$ é chamado ponto ejetivo de $A$ se existir uma vizinhança aberta $U_{0} \subset U$ de $x_{0}$ tal que $\forall x \in U_{0}-\left\{x_{0}\right\}$, existe um inteiro $m=m(x) \operatorname{com} A^{\jmath}(x)$ definida para $1 \leq \jmath \leq m$ e $A^{m}(x) \notin U_{0}$.

Lema 2.1. Sejam $S$ um subconjunto fechado convexo em um espaço de Banach $X, A$ : $S-\{0\} \rightarrow S$ completamente contínua, $0 \in S$ um ponto ejetivo de $A$ e assumamos que exista $N>0$ tal que $x \in S,\|x\|=N, A x=\lambda x \Rightarrow \lambda<1$. Então $A$ tem um ponto fixo $x_{0} \in S, 0<\left\|x_{0}\right\|<N$ se ou $S$ tem dimensão infinita ou 0 é um ponto extremo de $S$.

A seguir provaremos uma série de lemas que permitirão definir o operador de retorno já mencionado e finalmente conduzirão à ejetividade da origem. Muitas vezes os resultados serão mais fortes que os necessários, mas convenientes para o nosso objetivo no capítulo 4 .

Lema 2.2. Sejam $N>M, \alpha>0, \varphi \in \mathcal{C}$.Então $\exists t_{0} \geq 0$ tal que $x(t ; \alpha, \varphi) \in \vec{B}_{N}$ para $t \geq t_{0}$.

Demonstração: Da equação $(2.1-\alpha)$, segue que

$$
x(t) \cdot \frac{d x}{d t}(t)=-\alpha|x(t)|^{2}+\alpha F(x(t-1) \cdot x(t)
$$

onde "." significa o produto escalar do $\mathrm{R}^{2}$.A limitação da $F$ implica 


$$
\frac{d|x(t)|^{2}}{d t} \leq-\alpha|x(t)|[|x(t)|-M]^{*}, \quad t \geq 0
$$

Assim,

$$
\frac{d|x(t ; \alpha, \varphi)|}{d t} \leq-\alpha N[\dot{N}-M]<0
$$

sempre que $|x(t ; \alpha, \varphi)|>N$, oque implica na existência de algum $t_{0}=t_{0}(\alpha, \varphi, N) \geq 0$ tal que $x(t ; \alpha, \varphi) \in \bar{B}_{N}$ para $t \geq t_{0} \diamond \diamond$

Da demonstração do Lema 2.2 segue que se $x(t ; \alpha, \varphi)$ é solução periódica de $(2.1-\alpha)$ então $|x(t ; \alpha, \varphi)| \leq M, \forall t \in \mathbf{R}$.

É oportuno observarmos agora, que para o estudo da existência de soluções periódicas não triviais de $(2.1-\alpha)$, a hipótese $(H .2)$ pode ser substituida pela condição mais geral de existir uma bola fechada $\bar{B}_{M}$ invariante por $F$, isto é,

$(\overline{H .2})$ :

$$
F\left(\bar{B}_{M}\right) \subset \bar{B}_{M}
$$

De fato, se a função $F$ da equação $(2.1-\alpha)$ satisfaz $(\overline{H .2})$, definindo

$$
\bar{F}(x)=\left\{\begin{array}{ll}
F(x), & \text { se }|x| \leq M \\
F\left(M \frac{x}{|x|}\right), & \text { se }|x|>M
\end{array} .\right.
$$

segue que toda solução periódica $x(t)$ de $\dot{x}(t)=-\alpha x(t)+\alpha \bar{F}(x(t-1))$ deve satisfazer $|x(t)| \leq M$. Ela é , portanto, uma solução periódica de $(2.1-\alpha)$, com $F$ satisfazendo $(\overline{H .2})$.

Para o lema a seguir, definimos

$$
Q_{1}=\left\{x \in \mathbf{R}^{2} \mid x_{1} \geq 0, \quad x_{2} \geq 0\right\}, \quad Q_{2}=\left\{x \in \mathbf{R}^{2} \mid x_{1} \geq 0, \quad x_{2} \leq 0\right\}
$$

Lema 2.3. Consideremos a equação $(2.1-\alpha)$ com hipóteses $(H .1)$ e (H.2). Então para cada $\alpha>0$, existe uma função contínua $t_{1 \alpha}: K_{\alpha}-\{0\} \rightarrow[0, \infty)$ tal que $x_{1}\left(t_{1 \alpha}(\varphi) ; \alpha, \varphi\right)>$ 
$0, x\left(t_{1 \alpha}(\varphi) ; \alpha, \varphi\right) \in Q_{1}$ se $0 \leq t \leq t_{1 \alpha}(\varphi)$ e $x(t ; \alpha, \varphi) \in \operatorname{int}\left(Q_{2}\right)$ se $t_{1 \alpha}(\varphi)<t<t_{1 \alpha}(\varphi)+\delta$ para qualquer $\delta>0$ suficientemente pequeno.

Demonstração: Suponhamos que para algum $\alpha>0$ e alguma $\varphi \in K_{\alpha}-\{0\}$, não exista $t_{1} \geq 0$ tal que $x(t ; \alpha, \varphi)=x(t) \in Q_{1}$ para $0 \leq t \leq t_{1}, x_{1}\left(t_{1}\right)>0, x_{2}\left(t_{1}\right)=0$.

$\operatorname{De} \varphi=\left(\varphi_{1}, \varphi_{2}\right) \in I_{\alpha}-\{0\}$, segue que $\left(\varphi_{1}(0), \varphi_{2}(0)\right) \neq(0,0)$. É claro que $\varphi_{2}(0)>0$, caso contrário $t_{1}=0$.

Também, não há perda de generalidade em considerar $\varphi_{1}(0)>0$, se não, tomamos a condição inicial $\psi=x_{\tau}\left(\cdot ; \alpha, \varphi^{\prime}\right)$ para um conveniente $\tau>0$.

A equação $(2.1 . a-\alpha)$ e hipótese $(H .1)$ implicam $x_{1}(t) \geq \varphi_{1}(0) \epsilon^{-\alpha t}>0$ enquanto $x(\dot{t}-1)$ permanecer no primeiro quadrante $Q_{1} ;$ portanto $x(t ; \alpha, \varphi)$ não sai de $Q_{1}$ pelo eixo $x_{2}$.

De $(2.1 . b-\alpha)$ è $(H .1)$ segue que $\dot{x}_{2}(t) \leq-\alpha x_{2}(t) \leq 0$, ou seja, $x_{2}(t)$ é não crescente se $x(t) \in Q_{1}$; assim devemos ter

$$
\lim _{t \rightarrow \infty} x_{2}(t)=\eta \geq 0
$$

Se $\eta>0, \exists \bar{t}>0$ tal que $x_{2}(t)>\frac{\eta}{2}$ para $t \geq \bar{t}$ e então, por $(2.1 . b-\alpha)$,

$$
x_{2}(t) \leq x_{2}(\bar{t})-\frac{\alpha \eta}{2}(t-\bar{t}), \quad t \geq \bar{t}
$$

o que implica que $x_{2}(t)$ se torna negativa num tempo finito e isto contradiz o fato que $x(t) \in Q_{1},-1 \leq t<\infty$. Assim,

$$
\lim _{t \rightarrow \infty} x_{2}(t)=0
$$

Afirmamos que

$$
\lim _{t \rightarrow \infty} x_{1}(t)=0
$$

- Mostremos primeiramente que não podemos ter $x_{1}(t) \rightarrow \xi>0$ quando $t \rightarrow \infty$ :- como $F_{1}(\xi, 0)=0$, se $\lim _{t \rightarrow+\infty} x_{1}(t)=\xi>0$, segue de $(2.1 . a-\alpha)$ que $\lim _{t \rightarrow+\infty} \dot{x}_{1}(t)=-\alpha \xi<0$ 
assim, $x_{1}(t)$ fica negativa a partir de um tempo finito, e isso contradiz o fato de que $x(t) \in Q_{1},-1 \leq t<\infty$.

Pelo Lema 2.2, $\left|x_{1}(t)\right|<N$ para algum $N>0$. Suponhamos, por um momento, que (2.6) não seja verdade. Então $\exists \xi \in R$ tal que

$$
0 \leq \lim _{t \rightarrow \infty} \inf x_{1}(t)<\xi<\lim _{t \rightarrow \infty} \sup x_{1}(t) \leq N
$$

e uma sequência $\left(t_{n}\right) \rightarrow \infty, \operatorname{com} x_{1}\left(t_{n}\right)=\xi, \dot{x}_{1}\left(t_{n}\right) \geq 0$, o que é uma contradição já que por $(2.1 . a-\alpha)$,

$$
\dot{x}_{1}\left(t_{n}\right)=-\alpha \xi+\alpha F_{1}\left(x_{1}\left(t_{n-1}\right), x_{2}\left(t_{n-1}\right)\right) \rightarrow-\alpha \xi
$$

quando $t_{n}{ }^{\cdot} \rightarrow \infty$.

Assim vale (2.6) e portanto, sempre que $x(t)=x(t ; \alpha, \varphi)$ permanecer em $Q_{1}$ para $t \geq 0$

$$
\lim _{t \rightarrow \infty} x(t ; \alpha ; \varphi)=0
$$

Da hipótese (H.1) segue que a expansão de Taylor de $F_{2}(x)$ próximo à origem é

$$
F_{2}(x)=\delta_{2} x_{1}+x_{1}\left[a x_{1}+b x_{2}+O\left(|x|^{2}\right)\right]
$$

quando $x \rightarrow 0$, e isto implica que dado $0<\eta<-\delta_{2}$, existe uma vizinhança $\mathrm{V}$ do zero no $\mathbf{R}^{2}$ tal que se $x \in V, x_{1}>0$,

$$
F_{2}(x)<\left(\delta_{2}+\eta\right) x_{1}
$$

Por (2.7), não há perda de generalidade em assumir $x(t ; \alpha, \varphi) \in V \cap Q_{1}$, para $-1 \leq$ $t<\infty, x_{1}(-1)>0$, caso contrário tomamos como condição inicial $\psi=x_{\tau}(\cdot ; \alpha, \varphi)$ para $\tau>0$ suficientemente grande.

Como $(2.1 . b-\alpha)$ é equivalente a

$$
\begin{aligned}
& x_{2}(t)=\varphi_{2}(t), \quad t \in[-1,0] \\
& x_{2}(t)=x_{2}(0) \epsilon^{-\alpha t}+\alpha \int_{0}^{t} \epsilon^{\alpha(s-t)} F_{2}(x(s-1)) d s, t \geq 0
\end{aligned}
$$


segue por (2.8) que

$$
x_{2}(n+1)<x_{2}(0) e^{-\alpha(n+1)}+\alpha\left(\delta_{2}+\mu\right) e^{-\alpha n} \int_{0}^{n+1} e^{\alpha(s-1)} x_{1}(s-1) d s .
$$

Já que $\epsilon^{\alpha t} x_{1}(t)$ é não decrescente para $t \geq-1$,

$$
x_{2}(n+1)<e^{-\alpha(n+1)}\left[x_{2}(0)-(n+1) \alpha\left(-\delta_{2}-\mu\right) x_{1}(-1)\right]
$$

o que implica que $x_{2}(t)$ se torna negativa num tempo finito, uma contradição.

Portanto $\exists t_{1} \geq 0$ tal que

$$
x(t ; \alpha, \varphi) \in Q_{1} \text { para } 0 \leq t \leq t_{1}, \quad x_{1}\left(t_{1} ; \alpha, \varphi\right)>0, \quad x_{2}\left(t_{1} ; \alpha, \varphi\right)=0
$$

Se $\varphi_{2}(0)>0$ então $t_{1}>0$ e $x_{1}\left(t_{1}-1\right)>0$ tomando, se necessário, uma condição inicial $\psi=x_{\tau}(\cdot ; \alpha, \varphi)$ para um $\tau>0$ conveniente.

Assim, segue da equação $(2.1 . b-\alpha)$ que $x(t ; \alpha, \varphi)$ atravessa transversalmente o semieixo horizontal positivo no instante $t=t_{1}$ e definimos $t_{1 \alpha}(\varphi)=t_{1}$.

Se $\varphi_{2}(0)=0$ é fácil ver que existe $0 \leq \tau<1$ para o qual $x_{2}(t) \equiv 0, x_{1}(t)>0$ para $-1<t \leq \tau$. Neste caso, a solução $x(t ; \alpha, \varphi)$ deixa $Q_{1}$ no instante $t=\tau$, tangencialmente, e $t_{1 \alpha}(\varphi)=\tau$.

A continuidade de $t_{1 \alpha}$ segue agora do Teorema da Continuidade em relação às Condições Iniciais [9, cap 2, T. 2.2.]. $\diamond$

Lema 2.4. Seja $A \subset \mathcal{C}$ aberto, $0 \in A$. Existe uma vizinhança $V$ de 0 em $\mathcal{C}$ tal que $\forall \alpha>0, \forall \varphi \in V \cap K_{\alpha}, \varphi \neq 0$, tem-se $x_{t}(\cdot ; \alpha, \varphi) \in A$ para $0 \leq t \leq t_{1 \alpha}(\varphi)+1$.

Demonstração: É suficiente provar que dado $A \subset \mathbf{R}^{2}$ aberto, $0 \in A$, existe uma vizinhança $V$ de 0 em $\mathcal{C}$ tal que se $\alpha>0, \varphi \in V \cap K_{\alpha}, \varphi \neq 0$ então $x(t ; \alpha, \varphi) \in A$ para $-1 \leq t \leq t_{1 \alpha}(\varphi)+1$.

Admitindo que vale tal afirmação para $t \in\left[-1, t_{1 \alpha}(\varphi)\right]$, como $F$ é contínua, $F(0)=0$, segue das equações $(2.1 . a-\alpha),(2.1 . b-\alpha)$ e hipótese (H.1),

$$
\frac{d}{d t} e^{\alpha t} x_{1}(t) \leq \alpha e^{\alpha t} \varepsilon
$$




$$
\frac{d}{d t} e^{\alpha t} x_{2}(t) \geq-\alpha e^{\alpha t} \varepsilon
$$

com $t \in\left[t_{1 \alpha}(\varphi), t_{1 \alpha}(\varphi)+1\right]$, para qualquer $\varepsilon>0$, desde que $|x(t ; \alpha, \varphi)|$ seja suficientemente pequeno em $\left[-1, t_{1 \alpha}(\varphi)\right]$.

Essas desigualdades implicam que $0 \leq x_{1}(t ; \alpha, \varphi) \leq \varepsilon \mathrm{e}-\varepsilon \leq x_{2}(t ; \alpha, \varphi) \leq 0$ para $t \in\left[t_{1 \alpha}(\varphi), t_{1 \alpha}(\varphi)+1\right]$.

Suponhamos então que a afirmação não seja verdadeira para $t \in\left[-1, t_{1 \alpha}(\varphi)\right]$. Então existe retângulo aberto

$$
R=\left\{\left(x_{1}, x_{2}\right) \mid-\mu_{1}<x_{1}<l,-\mu_{2}<x_{2}<m\right\} \ni(0,0)
$$

tal que pára alguma sequência $\left(\alpha_{n}\right), \alpha_{n}>0$, e alguma sequência $\left(\varphi_{n}\right), \varphi_{n} \in \Lambda_{\alpha_{n}}-$ $\{0\}, \varphi_{n} \rightarrow 0$ quando $n \rightarrow+\infty, \exists\left(t_{n}\right),-1 \leq t_{n} \leq t_{1 \alpha_{n}}\left(\varphi_{n}\right) \operatorname{com} x\left(t_{n} ; a_{n}, \varphi_{n}\right) \in \partial R, n=$ $1,2, \ldots$

Sejam $x_{n}(t)=x\left(t ; \alpha_{n}, \varphi_{n}\right), x_{1 n}(t) \geq 0$ e $x_{2 n}(t) \geq 0$ enquanto $x_{n}(t)$ pertencer ao primeiro quadrante $Q_{1}$. Além disso, $x_{2 n}(t)$ é decrescente se $\varphi_{2 n}(0)>0$ ou $x_{2 n}(t) \equiv 0$ para $0 \leq t \leq t_{1 \alpha_{n}}\left(\varphi_{n}\right)$ se $\varphi_{2 n}(0)=0$.

Assim, $x_{n}(t) \in[0, l] \times\left[0, q_{n}\right],-1 \leq t \leq t_{n}$, onde $q_{n}=\left\|\varphi_{n}\right\| \rightarrow 0$ quando $n \rightarrow \infty$.

Consequentemente $x_{1}\left(t_{n} ; \alpha_{n}, \varphi_{n}\right)=l$ para $n=1,2, \ldots$

A continuidade de $F_{1}$ e $F_{1}\left(x_{1}, 0\right) \equiv 0$ implicam que $\lim _{x_{2} \rightarrow 0} F_{1}\left(x_{1}, x_{2}\right)=0$ uniformemente em $x_{1}, 0 \leq x_{1} \leq l$.

Assim podemos escrever, enquanto $x_{n}(t) \in[0, l] \times\left[0, q_{n}\right]$,

$$
x_{1 n}(t)=\varphi_{1 n}(0) e^{-\alpha_{n} t}+\alpha_{n} e^{-\alpha_{n} t} \int_{0}^{t} e^{\alpha_{n} s} F_{1}(x(s-1)) d s \leq q_{n} e^{-\alpha_{n} t}+M_{n}\left[1-e^{-\alpha_{n} t}\right]
$$

onde $M_{n}=\sup \left\{F_{1}(x) \mid x \in[0, l] \times\left[0, q_{n}\right]\right\}$.

Segue disto que $x_{1}\left(t_{n} ; a_{n}, \varphi_{n}\right)<q_{n}+M_{n}<l$ para $n$ suficientemente grande, o que contradiz a escolha de $\varphi_{n} \cdot \diamond$

Para $\alpha>0$, dada $\varphi \in K_{\alpha}^{-}-\{0\}$, seja $\psi=x_{t_{1 \alpha}(\varphi)+1}(\cdot ; \alpha, \varphi)$

$$
\left(e^{\alpha \theta} \dot{\psi}(\theta)\right)^{\prime}=\alpha \epsilon^{\alpha \theta} F\left(x\left(t_{1 \alpha}(\varphi)+\theta ; \alpha, \varphi\right)\right), \quad \theta \in[-1,0]
$$


Como $x\left(t_{1 \alpha}(\varphi)+\theta ; \alpha, \varphi\right) \in Q_{1}$ para $-1 \leq \theta \leq 0$ segue da hipótese $(H .1)$ que $\epsilon^{\alpha \theta_{\psi_{1}}(\theta)}$ é não decrescente e $\epsilon^{\alpha \theta} \psi_{2}(\theta)$ é não crescente.

Assim, se $\mathrm{R}$ é a rotação

$$
R=\left(\begin{array}{cc}
0 & 1 \\
-1 & 0
\end{array}\right)
$$

e $R K_{\alpha}:=\left\{R \varphi \mid \varphi \in K_{\alpha}\right\}$, essas propriedades de $\epsilon^{\alpha \theta} \psi(\theta)$ implicam que $\psi \in R I_{\alpha}$.

Podemos então, definir uma função

$$
\begin{aligned}
A_{1 \alpha}: K_{\alpha} & \longrightarrow R I_{\alpha} \\
A_{1 \alpha}(\varphi) & =x_{t_{1 \alpha}(\varphi)+1}(\cdot ; \alpha, \varphi), \varphi \neq 0 \\
A_{1 \alpha}(0) & =0
\end{aligned}
$$

Lema 2.5. Para cada $\alpha>0$, a função $A_{1 \alpha}: I_{\alpha} \rightarrow R I_{\alpha}$ é completamente contínua.

Demonstração: A continuidade de $t_{1 \alpha}$ e da solução de $(2.1-\alpha)$ em relação às condições iniciais garantem a continuidade de $A_{1 \alpha}$ em $I_{\alpha}-\{0\}$. Em $\varphi=0$ a continuidade segue do Lema 2.4.

Seja $L=\bar{D}_{\mu} \cap K_{\alpha}$. Então

$$
A_{1 \alpha}(L)=\left\{x_{t_{1 \alpha}(\varphi)+1}(\cdot ; \alpha, \varphi) \mid \varphi \in L-\{0\}\right\} \cup\{0\}
$$

Os mesmos argumentos da prova do Lema 2.2 implicam que $A_{1 \alpha}(L)$ é limitado por $\max \{M, \mu\}$.

A equicontinuidade segue agora da continuidade da função $H: \mathbf{R}^{2} \times \mathbf{R}^{2} \rightarrow \mathbf{R}^{2}$, definida por $H(u, v)=-\alpha u+\alpha F(v) \cdot \diamond$

A simetria rotacional advinda da hipótese (H.1) nos permite repetir os passos da construção de $A_{1 \alpha}$, juntamente com adaptações óbvias, para definir funçôes contínuas $\varphi \rightarrow t_{j \alpha}(\varphi)$ de $R^{j-1}\left(K_{\alpha}-\{0\}\right)$ em $[0,+\infty), j=2,3,4$ e dessas funções obtemos $A_{j \alpha}$ : $R^{j-1} K_{\alpha} \rightarrow R^{j} K_{\alpha}^{-}, j=2,3 ; 4$.

Definimos, então,

$$
\begin{aligned}
A_{\alpha}: I_{\alpha} & \longrightarrow R^{4} I_{\alpha}=I_{\alpha} \\
A_{\alpha}(\varphi) & =\left(A_{4 \alpha} \circ A_{3 \alpha} \circ A_{2 \alpha} \circ A_{1 \alpha}\right)(\varphi), \varphi \in I_{\alpha}-\{0\} \\
A_{\alpha}(0) & =0
\end{aligned}
$$


Assim, para cada $\varphi \in K_{\alpha}^{-}-\{0\}$, se chamarmos de $\tau_{\alpha}(\varphi)$ o instante para o qual $A_{\alpha}(\varphi)=x_{\tau_{\alpha}(\varphi)}(\cdot ; \alpha, \varphi)$, segue que

$$
\tau_{\alpha}(\varphi)=t_{1 \alpha}(\varphi)+t_{2 \alpha}\left(A_{1 \alpha} \varphi\right)+t_{3 \alpha}\left(A_{2 \alpha} A_{1 \alpha} \varphi\right)+t_{4 \alpha}\left(A_{3 \alpha} A_{2 \alpha} A_{1 \alpha} \varphi\right)+4
$$

O último termo "4" aparece nesta expressão èm virtude do efeito do retardamento para cada cruzamento da órbita com os eixos coordenados.

Da expressão (2.10) segue a continuidade da função

$$
\tau_{\alpha}: I_{\alpha}-\{0\} \rightarrow[4, \infty)
$$

Esses resultados sugerem a figura 2.1 a seguir.

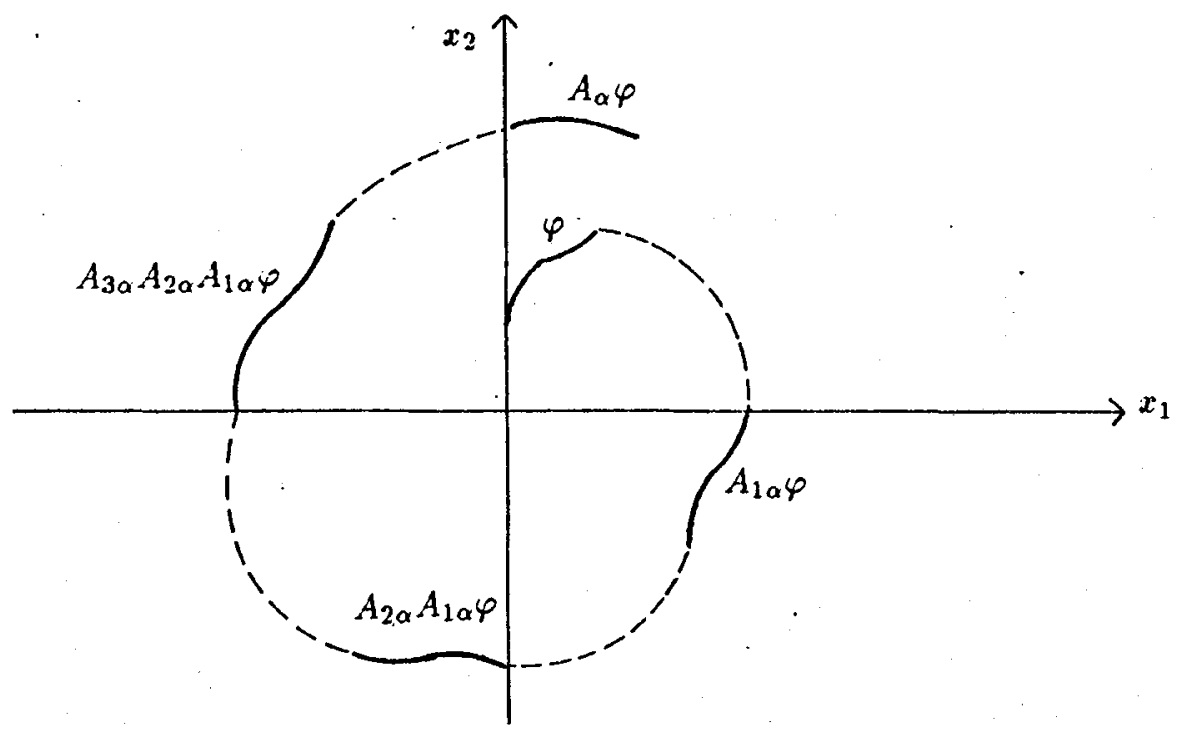

Figura 2.1

Observemos que, da definição de $t_{1 \alpha}$ no Lema 2.3 , a função $\psi=x_{t_{1 \alpha}(\varphi)+1}(\cdot ; \alpha, \varphi)$ é tal que $\psi_{1}(\theta)>0$ para $-1 \leq \theta \leq 0, \psi_{2}(-1)=0, \psi_{2}(\theta)<0$ para $-1<\theta \leq 0$. Isto garante que a solução $x(t ; \alpha, \varphi)$, com condição inicial $\varphi$ em $\Pi_{\alpha}$, caminhando no sentido horário no plano fase $x_{1} x_{2}$, corta transversalmente os demais semi-eixos nos instantes $t_{2 \alpha}\left(A_{1 \alpha}\right)+t_{1 \alpha}(\varphi)+1, \quad t_{3 \alpha}\left(A_{2 \alpha} A_{1 \alpha} \varphi\right)+t_{2 \alpha}\left(A_{1 \alpha} \varphi\right)+t_{1 \alpha}(\varphi)+2, \quad t_{4 \alpha}\left(A_{3 \alpha} A_{2 \alpha} A_{1 \alpha} \varphi\right)+$ $t_{3 \alpha}\left(A_{2 \alpha} A_{1 \alpha} \varphi\right)+t_{2 \alpha}\left(A_{1 \alpha} \varphi\right)+t_{1 \alpha}(\varphi)+3$.

Assim, podemos adiantar que se $x(\cdot ; \alpha, \varphi)$ é solução periódica não trivial de $(2.1-\alpha)$, obtida através de um ponto fixo de $A_{\alpha}$, então $\varphi_{1}(\theta)>0$ para $-1<\theta \leq 0, \varphi_{2}(\theta)>0$. 
O lema que segue é consequência imediata do Lema 2.4 e das simetrias das nossas hipóteses.

Lema 2.6. Seja $A$ um subconjunto aberto de $\mathcal{C}, 0 \in A$. Existe uma vizinhança $V$ de 0 em $\mathcal{C}$ tal que se $\alpha>0, \varphi \in V \cap \kappa_{\alpha}, \varphi \neq 0$ então $x_{t}(\cdot ; \alpha, \varphi) \in A$ para $0 \leq t \leq \tau_{\alpha}(\varphi)$.

Lema 2.7. Para cada $\alpha>0$, existe um conjunto fechado convexo $I_{\alpha} \subset \mathcal{C}, 0 \in I_{\alpha}$, e uma função continua $\tau_{\alpha}: K_{\alpha}-\{0\} \rightarrow[4, \infty)$ tal que a função

$$
\begin{aligned}
A_{\alpha}: \bar{K}_{\alpha} & \longrightarrow \mathcal{C} \\
A_{\alpha}(\varphi) & =x_{\tau_{\alpha}(\varphi)}(\cdot ; \alpha, \varphi), \varphi \in K_{\alpha}-\{0\} \\
A_{\alpha}(0) & =0
\end{aligned}
$$

é completamente contínua e $A_{\alpha} \Pi_{\alpha} \subset H_{\alpha}^{\circ}$.

Demonstração: consequência imediata do Lema 2.5 e observações após a sua demonstração. $\diamond$

Precisamos agora relembrar a decomposição do espaço fase como uma soma direta, $\mathcal{C}=P_{\lambda} \oplus Q_{\lambda}$, associada a um autovalor $\lambda$ da equação característica [9,cap 7$]$.

Linearizando $(2.1-\alpha)$ em torno da solução nula segue

$$
\dot{y}(t)=\alpha L\left(y_{t}\right)
$$

onde $L: \mathcal{C} \rightarrow \mathbf{R}^{2}, L(\varphi)=-\varphi(0)+B \varphi(-1) \mathrm{e}$

$$
B:=\left(\begin{array}{cc}
0 & \delta_{1} \\
\delta_{2} & 0
\end{array}\right)
$$

Seja $\eta(\theta), \theta \in[-1,0]$, matriz de ordem 2 definida por

$$
\eta(\theta)=\left\{\begin{array}{cl}
0, & \theta=-1 \\
B, & -1<\theta<0 \\
-I+B, & \theta=0
\end{array}\right.
$$

Então para qualquer $\varphi \in \mathcal{C}$,

$$
L(\varphi)=\int_{-1}^{0}[d \eta(\theta)] \varphi(\theta)
$$


Seja $G_{\alpha}$ o gerador infinitesimal do semigrupo $\left\{T_{\alpha}(t), t \geq 0\right\}$ definido pelas soluções de $(2.11-\alpha)$,

$$
T_{\alpha}(t) \varphi=y_{t}(\cdot ; \alpha, \varphi), \quad \varphi \in \mathcal{C}, t \geq 0
$$

Seja $\alpha_{0} \in(0, \infty)$ dado em (1.7). Conforme análise feita no Capítulo 1 , para cada $\alpha>\alpha_{0}, G_{\alpha}$ tem um único autovalor $\lambda=\lambda(\alpha) \in S_{0}=\left\{\lambda \in \mathbf{C} \mid 0<\Im \lambda<\frac{\pi}{2}\right\}$ com $\Re \lambda(\alpha)>0$ e tal autovalor é simples.

Assim, $M_{\lambda}\left(G_{\alpha}\right)=\mathcal{N}\left(\lambda I-G_{\alpha}\right)$, o auto espaço generalizado associado a $\lambda$, é 1dimensional, e o chamaremos de $M\left(G_{\alpha}\right)$ ou, mais simplesmente, de $P_{\alpha}$, a fim de simplificar notações.

Seja $\mathcal{C}^{\star}=\mathcal{C}\left([0,1], \mathbf{R}^{2^{\star}}\right)$, onde $\mathbf{R}^{\mathbf{2}^{\star}}$ é o plano dos. vetores linhas.

A forma bilinear de $\mathcal{C}^{\star} \times \mathcal{C}$, dada por

$$
\left\langle\psi, \varphi>=\psi(0) \cdot \varphi(0)-\int_{-1}^{0} \int_{0}^{\theta} \psi(\xi-\theta)[d \eta(\theta)] \varphi(\xi) d \xi\right.
$$

$\psi \in \mathcal{C}^{\star}, \varphi \in \mathcal{C}$, "." significando produto escalar do $\mathbf{R}^{2}$, aparece de maneira natural na decomposição de $\mathcal{C}$.

Se $G_{\alpha}^{\star}$ é o operador adjunto formal de $G_{\alpha}, \lambda$ é também autovalor de $G^{\star}$ e seu auto espaço generalizado $M\left(G_{\alpha}^{\star}\right)$ é 1-dimensional.

Se $\mu$ é uma solução não trivial de $H_{\alpha}(\lambda) \mu=0 \operatorname{com} H_{\alpha}(\lambda)=(\lambda+\alpha) I-\alpha B \epsilon^{-\lambda}$ dado em $(1.3-\alpha)$, e o vetor linha $v$ é uma solução não trivial de $v H_{\alpha}(\lambda)=0$ então as funções $\rho_{\alpha}(\theta)=e^{\lambda \theta} \mu, \theta \in[-1,0]$, e $\sigma_{\alpha}(s)=v e^{-\lambda s}, s \in[0,1]$, geram $M\left(G_{\alpha}\right)$ e $M\left(G_{\alpha}^{*}\right)$, respectivamente.

Existem $u$ e $v$ convenientes de modo que, dada $\varphi \in \mathcal{C}$, a componente $\varphi_{\alpha}$ pertencente. a $P_{\alpha}$, obtida da decomposição de $\mathcal{C}$ em $P_{\alpha} \oplus Q_{\alpha}$, é tal que

$$
\pi_{\alpha}(\varphi):=\varphi_{\alpha}=<\sigma_{\alpha}, \varphi>\rho_{\alpha}
$$

Voltamos a enfatizar que a afirmação dada no lema a seguir é mais forte do que o necessário neste capítulo, no que se refere à dependência do parâmetro $\alpha$. 
Lema 2.8. Para qualquer compacto $J_{0} \subset\left(\alpha_{0}, \infty\right)$, e qualquer constante $a, 0<a<M$, $\Upsilon=\inf \left\{\left\|\pi_{\alpha} x_{t}\right\| \mid x_{t}=x_{t}(\cdot ; \alpha, \varphi), \alpha \in J_{0}, \varphi \in K_{\alpha},\|\dot{\varphi}\|=a, 0 \leq t \leq \tau_{\alpha}(\varphi)\right\}>0$.

A prova deste lema consiste da parte mais trabalhosa deste capítulo e segue dos quatro lemas subsequentes.

Dado um compacto $J_{0} \subset(0, \infty)$, chamaremos de $\alpha_{1}=\min J_{0}, \alpha_{2}=\max J_{0}$ e observemos que se $\alpha \in J_{0}, \varphi \in K_{\alpha}$ então valem

(i) $\phi_{j}(\theta) \leq \epsilon^{\alpha_{2}} \phi_{j}(s), \quad j=1,2, \quad-1 \leq \theta \leq s \leq 0$.

(ii) $\|\phi\| \geq d \Rightarrow|\phi(0)| \geq d \epsilon^{-\alpha_{2}}$.

Os três próximos lemas descrevem o comportamento da solução $x(\cdot ; \alpha, \varphi)$ de $(2.1-\alpha)$ com $\alpha \in J_{0}, \varphi \in K_{\alpha},\|\varphi\| \geq d$. É nosso propósito obter uma estimativa inferior independente do parâmetro a para $x_{1}\left(t_{1 \alpha}(\varphi) ; \alpha, \varphi\right)$, com $t_{1 \alpha}$ definido no Lema 2.3.

A figura seguinte, $\operatorname{com} k$ e $\sigma$ suficientemente pequenos, ilustra a evolução, em $Q_{1}$, de uma solução sob tais condições e pode ser útil ao leitor no entendimento dos passos das provas.

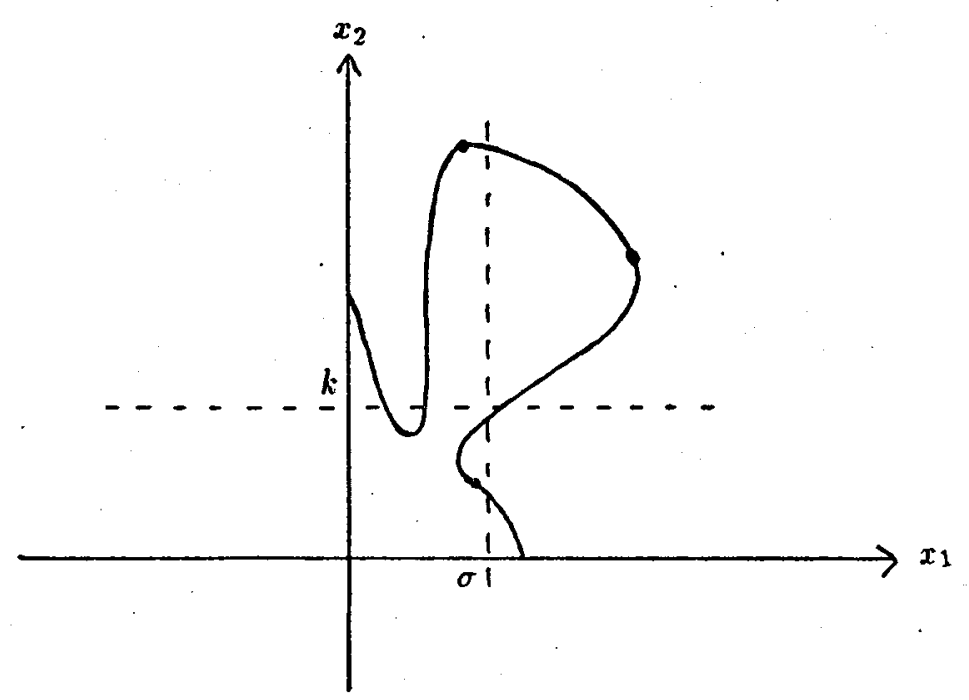

Figura 2.2

Lema 2.9. Sejam $d>0$ e $J_{0}$ um compacto de $(0, \infty)$. Então $\exists k>0, \sigma>0$ tais que $\forall \alpha \in$ 
Demonstração: Da hipótese (H.1) segue que a expansão de Taylor de $F_{2}(x)$ perto da origem é

$$
F_{2}(x)=\delta_{2} x_{1}+x_{1}\left[a x_{1}+b x_{2}+O\left(|x|^{2}\right)\right]
$$

quando $x \rightarrow 0$.

Assim, dado $0<\eta<-\delta_{2}$, existe vizinhança $\mathrm{V}$ de 0 em $\mathbf{R}^{2}$ tal que se $x \in V, x_{1}>0$

$$
F_{2}(x)<\left(\delta_{2}+\eta\right) x_{1}
$$

Seja $\xi$ tão pequeno de modo que o retângulo $R=\left\{\left(x_{1}, x_{2}\right) \mid-\xi<x_{1}<\xi,-\xi<x_{2}<\right.$ $\xi$ \} esteja contido em V.

Definimos:

$$
\cdot k:=\min \left\{\frac{d}{4 e^{3 \alpha_{2}}}, \xi\right\} .
$$

Também, por (H.1), valem as afirmações:

$$
\begin{gathered}
\exists \sigma^{\prime}>0 \mid\left(x_{1}, x_{2}\right) \in Q_{1}, \quad x_{2}>k \Longrightarrow F_{1}\left(x_{1}, x_{2}\right)>\max \left\{\sigma^{\prime}, \frac{\sigma^{\prime}}{\alpha_{1}}\right\} \\
\forall \varepsilon>0, \varepsilon<\frac{d}{4 e^{3 \alpha_{2}}}, \quad \exists \delta=\delta(\varepsilon)>0 \mid\left(x_{1}, x_{2}\right) \in Q_{1}, x_{1} \leq \delta(\varepsilon) . \\
\Longrightarrow-\varepsilon \leq F_{2}\left(x_{1}, x_{2}\right)<0 .
\end{gathered}
$$

Seja:

$$
\sigma:=\min \left\{\frac{\delta(\xi)}{e^{\alpha_{2}}}, \frac{\sigma^{\prime}}{2}, k\right\} .
$$

Segue de (2.16), (2.17) e da equação $(2.1 . b-\alpha)$,

$$
x_{2}(t ; \alpha, \varphi) \geq\left[\varphi_{2}(0)+\varepsilon\right] e^{-\alpha t}-\varepsilon, \quad t \in[0,1]
$$

Além disso $\varphi_{2}(0) \geq \frac{d}{2 e^{\alpha_{2}}}$ pois $\varphi_{1}(0) \leq \sigma,\|\varphi\| \geq d$ e assim 


$$
x_{2}(t ; \alpha, \varphi)>k, \quad t \in[0, \dot{1}]
$$

já que $E(t):=\left[\frac{d}{2 e^{\alpha_{2}}}+\varepsilon\right] \epsilon^{-\alpha t}-\varepsilon$ é uma função decrescente em $[0,1]$ e $E(1)>\frac{d}{4 e^{2 \alpha_{2}}}$.

O Lema 2.3 garante a existência de um primeiro instante $\hat{t}$ para o qual $x_{2}(\hat{t} ; \alpha, \varphi)=k$ e $x_{2}(t ; \alpha, \varphi)>k$ para $t<\hat{t}$.

Suponhamos por um momento que $x_{1}(t) \leq \sigma$ para todo $t \in[0, \hat{t}]$. Então $x_{1}(t-1) \leq \sigma e^{\alpha_{2}}, \quad t \in[0, \hat{t}]$.

Repetindo o argumento anterior segue que

$$
x_{2}(t ; \alpha, \varphi) \geq E(t), t \in[0, \widehat{t}]
$$

Como $E(2)>\frac{d}{4 e^{3 a_{2}}}>k$, temos que $\widehat{t}>2$.

Por $(2.1 . a-\alpha)$ e (2.15) segue agora, para $t \in[1,2]$,

$$
\dot{x}_{1}(t) \geq-\alpha \sigma+\alpha \max \left\{\sigma^{\prime}, \frac{\sigma^{\prime}}{\alpha_{1}}\right\} \geq-\alpha \sigma+\max \{2 \alpha \sigma, 2 \sigma\} \geq \sigma .
$$

Assim, $\exists t \in(1,2)$ para o qual $x_{1}(t)>\sigma$, uma contradição .

Isto garante a existência de $\tau_{1} \in(0, \hat{t}]$, de acordo com o enunciado. $\diamond$

Lema 2.10. Admitamos as hipóteses do Lema 2.9 e sejam $k$ e $\sigma$ definidos em (2.14) e (2.17), respectivamente. Se, para $\alpha \in J_{0}, \varphi \in K_{\alpha}$, a solução $x(t ; \alpha, \varphi)$ de $(2.1-\alpha)$ satisfaz $x_{1}\left(\tau_{1} ; \alpha, \varphi\right)>\sigma, x_{2}\left(\tau_{1} ; \alpha, \varphi\right) \geq k$ para algum $\tau_{1} \geq 0$, então $\exists \tau_{2}=\tau_{2}(\alpha, \varphi)>0$ tal que $x_{1}(t ; \alpha, \varphi)>\sigma e^{-\alpha_{2}}$ para $t \in\left[\tau_{1}, \tau_{2}\right], 0<x_{2}\left(\tau_{2} ; \alpha, \varphi\right)<k$.

Demonstração: Como $x_{1}\left(\tau_{1} ; \alpha, \varphi\right)>\sigma$, segue de $(2.1 . a-\alpha)$ que

$$
x_{1}(t ; \alpha, \varphi)>\sigma e^{-\alpha_{2}}, \quad t \in\left[\tau_{1}, \tau_{1}+1\right]
$$

Se $0<x_{2}(t ; \alpha, \varphi)<k$ para algum $t \in\left[\tau_{1}, \tau_{1}+1\right]$, definimos $\tau_{2}$ como sendo tal $t$.

Suponhamos, então, que $x_{2}(t ; \alpha, \varphi)>k$ para todo $t \in\left[\tau_{1}, \tau_{1}+1\right]$.

Seja $\tilde{t}=\tilde{t}(\alpha, \varphi)>\tau_{1}+1$ o instante $\mathrm{t}$ para o qual $x_{2}(t ; \alpha, \varphi)=k$ e $x_{2}(t+\varepsilon ; \alpha, \varphi)<$ $k, \forall \varepsilon>0$. 
A equação (2.1.a $-\alpha)$ e as expressões (2.15) e (2.17) implicam que

$$
\dot{x}_{1}(t) \geq-\alpha x_{1}(t)+\alpha \sigma e^{-\alpha_{2}}, \quad t \in\left[\tau_{1}+1, \tilde{t}+1\right]
$$

e então

$$
x_{1}(t)>\sigma e^{-\alpha_{2}}, \quad \forall t \in\left[\tau_{1}+1, \tilde{t}+1\right] .
$$

Consequentemente, existe $\tau_{2}$. de acordo com o enunciado. $\diamond$

Lema 2.11. Admitamos as hipóteses do Lema 2.9 e sejam $k$ e $\sigma$ definidos em (2.14) e (2.17), respectivamente. Então $\exists c>0$ tal que para $\alpha \in J_{0}, \varphi \in K_{\alpha}$ e $x(t ; \alpha, \varphi)$ solução de $(2.1-\alpha)$ satisfazendo $x_{1}\left(\tau_{2} ; \alpha, \varphi\right)>\sigma \epsilon^{-\alpha_{2}}, 0<x_{2}\left(\tau_{2} ; \alpha, \varphi\right)<k$ para algum $\tau_{2} \geq 0$ se tem $x_{1}\left(t_{1} ; \alpha, \varphi\right) \geq c$, com $t_{1}=t_{1 \alpha}(\varphi)$ definido no Lema 2.3 .

Demonstração: $\operatorname{Se} x_{1}(t ; \alpha, \varphi)>\sigma e^{-\alpha_{2}}$ em algum intervalo $\left(t_{1}-\varepsilon, t_{1}\right)$ definimos $c:=$ $\sigma e^{-\alpha_{2}}$

Suponhamos, então, que isto não aconteça e seja $\bar{t}=\bar{t}(\alpha, \varphi), \tau_{2}<\bar{t}<t_{1}$ o último instante $t$ para o qual $x_{1}(t ; \alpha, \varphi)=\sigma e^{-\alpha_{2}}$.

Admitindo $t_{1}>\bar{t}+1$, segue da equação $(2.1 . b-\alpha)$ e de (2.13),

$$
x_{2}(t ; \alpha, \varphi) \leq e^{-\alpha(t-\bar{t}-1)} x_{2}(\bar{t}+1)+\alpha\left(\delta_{2}+\eta\right) e^{-\alpha t} \int_{\bar{t}+1}^{t} e^{\alpha s} x_{1}(s-1 ; \alpha, \varphi) d s
$$

para $t \in\left[\bar{t}+1, t_{1}+1\right]$.

Como $\epsilon^{\alpha t} x_{1}(t)$ é monótona crescente em $Q_{1}$,

$$
x_{2}(t ; \alpha, \varphi) \leq e^{-\alpha(t-\bar{t}-1)}\left[k+\alpha_{1}\left(\delta_{2}+\eta\right) \sigma e^{-\alpha_{2}}(t-\bar{t}-1)\right]
$$

O segundo membro desta desigualdade se anula no instante $\tau$ definido por

$$
\dot{\tau}=\frac{K e^{\alpha_{2}}}{\alpha_{1}\left(-\delta_{2}-\eta\right) \sigma}+\bar{t}+1
$$

Da equação $(2.1 . a-\alpha)$, 


$$
x_{1}(t ; \alpha, \varphi) \geq \sigma e^{-\alpha_{2}} e^{-\alpha(t-\bar{t})}, \quad i \in\left[\bar{t}, t_{1}+1\right]
$$

Como $t_{1} \leq \tau$, segue de (2.18) e (2.19) que $x_{1}\left(t_{1} ; \alpha, \varphi\right) \geq c$ onde

$$
c:=\sigma e^{\left(-2+\frac{k e^{\alpha_{2}}}{\alpha_{1}\left(\delta_{2}+\eta\right) \sigma}\right) \alpha_{2}} .
$$

Se $t_{1} \leq \bar{t}+1$, a desigualdade (2.19) implica que

$$
x_{1}(t ; \alpha, \varphi) \geq \sigma \epsilon^{-2 \alpha_{2}}
$$

Lema 2.12. Sejam $d>0$ e $J_{0}$ um compacto de $(0, \infty)$. Existe $c>0$ tal que se $\alpha \in$ $J_{0}, \varphi \in I_{\alpha},\|\varphi\| \geq d$, então $x_{1}\left(t_{1} ; \alpha, \varphi\right) \geq c$ e $x_{1}(t ; \alpha, \varphi) \geq c e^{-\alpha_{2}}$ para $t \in\left[t_{1}, t_{1}+1\right]$, com $t_{1}=t_{1 \alpha}(\varphi) \geq 0$ definido no Lema 2.3 .

Demonstração: Basta provar a primeira parte do Lema, já que a segunda segue trivialmente da primeira e da equaçào (2.1.a $a)$.

Se $\varphi_{2}(0)>0$, é consequência dos Lemas $2.9,2.10$ e 2.11 .

Se $\varphi_{2}(0)=0$ então $\varphi_{1}(0) \geq d \epsilon^{-\alpha_{2}}$, já que $\|\varphi\| \geq d$.

Além disso, $t_{1} \in[0,1)$ e por $(2.1 . a-\alpha)$,

$$
x_{1}\left(t_{1} ; \alpha, \varphi\right) \geq d e^{-2 \alpha_{2}}
$$

O lema seguinte é o resultado definitivo para a demonstração do Lema 2.8 .

Lema 2.13. Para qualquer constante real $d, 0<d<M$, e qualquer subconjunto compacto $J_{0}$ de $\left(\alpha_{0}, \infty\right)$

$$
\begin{array}{r}
m=\inf \left\{\mid<\sigma_{\alpha}, x_{t}>\left\|x_{t}=x_{t}(\cdot ; \alpha, \varphi), \varphi \in K_{\alpha}, d \leq\right\| \varphi \| \leq M,\right. \\
\left.\alpha \in J_{0}, 0 \leq t \leq t_{1 \alpha}(\varphi)+1\right\}>0 .
\end{array}
$$

Demonstração: Tomando 


$$
\sigma_{\alpha}(s)=(1,-i \mu) \epsilon^{-\lambda s}, \quad \mu=\sqrt{\frac{-\delta_{1}}{\delta_{2}}}, s \in[0,1]
$$

segue de (2.12),

$$
<\sigma_{\alpha}, x_{t}>=x_{1}(t)-i \mu x_{2}(t)+\alpha \int_{-1}^{0} e^{-\lambda(\xi+1)}\left[\delta_{1} x_{2}(t+\xi)-i \delta_{2} \mu x_{1}(t+\xi)\right] d \xi
$$

* Suponhamos $m=0$. Então existem sequências

$$
\alpha_{n} \in J_{0}, \quad \varphi_{n} \in K_{\alpha_{n}}, \quad d \leq\left\|\varphi_{n}\right\| \leq M, \quad t_{n} \in\left[0, t_{1 \alpha_{n}}\left(\varphi_{n}\right)+1\right], \quad n=1,2, \ldots
$$

tais que se $x\left(t ; \alpha_{n}, \varphi_{n}\right)=x_{n}(t)=\left(x_{1 n}(t), x_{2 n}(t)\right), \lambda_{n}=\lambda\left(\alpha_{n}\right)$,

$$
\begin{aligned}
<\sigma_{\alpha}, x_{n t_{n}}>= & x_{1 n}\left(t_{n}\right)-i \mu x_{2 n}\left(t_{n}\right)+ \\
& \quad \alpha_{n} \int_{-1}^{0} e^{-\lambda_{n}(\xi+1)}\left[\delta_{1} x_{2 n}\left(t_{n}+\xi\right)-i \delta_{2} \mu x_{1 n}\left(t_{n}+\xi\right)\right] d \xi
\end{aligned}
$$

tende a zero, quando $n$ tende a $\infty$.

Tomando $\lambda_{n}=a_{n}+b_{n} i$ com $a_{n}>0,0<b_{n}<\frac{\pi}{2}$, a expressão acima é equivalente a

$$
\begin{aligned}
<\sigma_{\alpha} ; x_{n t_{n}}>= & x_{1 n}\left(t_{n}\right)+\alpha_{n} \int_{-1}^{0} e^{-a_{n}(\xi+1)}\left[\delta_{1} x_{2 n}\left(t_{n}+\xi\right) \cos b_{n}(\xi+1)-\right. \\
& \left.-\delta_{2} \mu x_{1 n}\left(t_{n}+\xi\right) \operatorname{sen} b_{n}(\xi+1)\right] d \xi+i\left\{-\mu x_{2 n}\left(t_{n}\right)+\right. \\
& +\alpha_{n} \int_{-1}^{0} e^{-a_{n}(\xi+1)}\left[-\delta_{2} \mu x_{1 n}\left(t_{n}+\xi\right) \cos b_{n}(\xi+1)-\right. \\
& \left.\left.-\delta_{1} x_{2 n}\left(t_{n}+\xi\right) \operatorname{sen} b_{n}(\xi+.1)\right] d \xi\right\}
\end{aligned}
$$

Como $d \leq\left\|\varphi_{n}\right\| \leq M$, segue da prova do Lema 2.2 que $\left|x_{n}(t)\right| \leq M$ para $t \geq-1$ e para qualquer $n$.

Assim, $x_{n}\left(t_{n}\right)$ é convergente, tomando uma subsequência, se necessário. Também, como $\alpha_{n} \in J_{0}$ compacto, podemos tomar $\alpha_{n} \rightarrow \alpha$.

Seja $\left(L_{1}, L_{2}\right)=\lim _{n \rightarrow \infty}\left(x_{1 n}\left(t_{n}\right), x_{2 n}\left(t_{n}\right)\right)$.

Pelos Lemas 2.9 a 2.12, podemos afirmar que $L_{1}$ e $L_{2}$ não são simultaneamente nulos. 
Se $L_{1}>0, L_{2} \geq 0, \Re<\sigma, x_{n t_{n}}>$ fica positiva e longe do zero para $n$ suficientemente grande e se $L_{1}=0, L_{2}>0, \Im<\sigma, x_{n t_{n}}>$ fica negativa e longe do zero para $n$ suficientemente grande, o que é uma contradição, em ambos os casos.

Analisemos, então, o caso $L_{2}<0$.

É claro que $L_{1}>0$ já que, para $n$ suficientemente grande, $t_{1 \alpha_{n}}\left(\varphi_{n}\right) \leq t_{n} \leq t_{1 \alpha_{n}}\left(\varphi_{n}\right)+1$ e pelo Lema $2.12, x_{1}\left(t_{n} ; \alpha_{n}, \varphi_{n}\right) \geq c \epsilon^{-\alpha_{2}}$.

Assim, na expressão (2.20), para $n$ suficientemente grande,

$$
\begin{gathered}
x_{1 n}\left(t_{n}\right)-i \mu x_{2 n}\left(t_{n}\right)>\frac{L_{1}}{2}-i \mu \frac{L_{2}}{2} \\
p_{n}(\xi)=\delta_{1} x_{2 n}\left(t_{n}+\xi\right)-i \delta_{2} \mu x_{1 n}\left(t_{n}+\xi\right), \quad \xi \in[-1,0]
\end{gathered}
$$

é uma curva no plano complexo no setor $r \epsilon^{i \theta} \operatorname{com} 0 \leq \theta \leq \pi, r \geq 0$ e $e^{-\lambda_{n}(\xi+1)}, \xi \in[-1,0]$, está situada no setor $z=r \epsilon^{i} \theta$ com $r \geq \frac{1}{\delta}$ e $\frac{\pi}{2}<-B \leq \theta \leq 0$ para algum $B \in\left(0, \frac{\pi}{2}\right)$.

$\mathrm{O}$ integrando $e^{-\lambda_{n}(\xi+1)} p_{n}(\xi)$ tem argumento $\theta_{n}(\xi), \frac{-\pi}{2}<-B \leq \theta_{n}(\xi) \leq \pi$.

Tomando

$$
\tilde{\theta}_{n}=\max \left\{\arg e^{-\lambda_{n}(\xi+1)} p_{n}(\xi) \mid \xi \in[-1,0]\right\}
$$

prova-se que a curva $e^{-\lambda(\xi+1)} p_{n}(\xi), \xi \in[-1,0]$, está situada inteiramente no semiplano determinado pela reta com coeficiente $\tilde{\theta}_{n}$, o qual contém o semi-eixo x positivo.

Se $0 \leq \widetilde{\theta}_{n} \leq \frac{\pi}{2}$, então

$$
<\sigma, x_{t_{n}}>\not \rightarrow 0
$$

já que $e^{-\lambda_{n}(\xi+1)} p_{n}(\xi)$ tem parte real não negativa e vale (2.21).

Se $\frac{\pi}{2}<\tilde{\theta}_{n}<\pi$, definindo $\omega_{n}=\frac{\pi}{2}-\tilde{\theta}_{n}$, o mesmo argumento anterior implica que

$$
e^{i \omega_{n}}<\sigma, x_{t_{n}}>\nrightarrow 0
$$

do que segue o reultado. $\diamond$ 
Observemos que vale a mesma afirmação do Lema 2.13 se substituirmos $K_{\alpha}$ pela s rotações $R^{\jmath} \Lambda_{\alpha}^{\circ}$ e o intervalo $\left[0, t_{1 \alpha}(\varphi)+1\right]$ por $\left[t_{\jmath \alpha}(\varphi) \dot{+} 1, t_{(\jmath+1) \alpha}(\varphi)+1\right], \quad \jmath=1,2,3$, respectivamente, o que é justificado pela simetria rotacional.

Demostração do Lema 2.8: Seja $0<a<M$.

O Lema 2.13 implica que $\inf \left\{\left|<\sigma_{\alpha}, x_{t}>\right| \mid x_{t}=x_{t}(. ; \alpha, \varphi), \alpha \in J_{0}, \varphi \in K_{\alpha},\|\varphi\|=a, 0 \leq t \leq t_{1 \alpha}(\varphi)+1\right\}>0$.

Pelo Lema 2.12, sabemos que $\exists d_{1}>0$ tal que

$$
\left\|x_{t_{1 \alpha}(\varphi)+1}(\cdot ; \alpha, \varphi)\right\| \geq d_{1}, \quad \forall \alpha \in J_{0}, \varphi \in K_{\alpha},\|\varphi\|=a
$$

Além disso, pelo Lema 2.2,

$$
M \geq\left\|x_{t_{1 \alpha}(\varphi)+1}(. ; \alpha, \varphi)\right\|, \quad \forall \alpha \in J_{0}, \quad \varphi \in I_{\alpha}, \quad\|\varphi\|=a .
$$

Então, pelo Lema 2.8, $\inf \left\{\left|<\sigma_{\alpha}, x_{t}\right\rangle|| x_{t}=x_{t}(\cdot ; \alpha, \varphi), \alpha \in J_{0}, \varphi \in I_{\alpha},\|\varphi\|=a, 0 \leq t \leq t_{2 \alpha}(\varphi)+1\right\}>0$

$O$ resultado segue por procedimento análogo nos intervalos $\left[t_{2 \alpha}(\varphi)+1, t_{3 \alpha}(\varphi)+\right.$ $1],\left[t_{3 \alpha}(\varphi)+1, \tau_{\alpha}(\varphi)\right] . \diamond$

Lema 2.14. Suponhamos que as seguintes condições estejam satisfeitas:

(i) Existe um autovalor $\lambda$ de $(2.11-\alpha) \operatorname{com} \Re \lambda>0$.

(ii) Dados $\alpha>\alpha_{0}, G \subset \mathcal{C}$ aberto, $0 \in G$, existe uma vizinhança $V$ de 0 tal que $x_{t}(\cdot ; \alpha, \varphi) \in$ $G$ se $\varphi \in V \cap K_{\alpha}, \varphi \neq 0,0 \leq t \leq \tau_{\alpha}(\varphi)$.

(iii) Existe subconjunto $I_{\alpha}$ convexo e fechado de $\mathcal{C}, 0 \in K_{\alpha}$, e uma função contínua

$$
\tau_{\alpha}: K_{\alpha}-\{0\} \rightarrow[4, \infty)
$$

tal que a função

$$
\begin{aligned}
A_{\alpha}: I_{\alpha} & \longrightarrow C \\
A_{\alpha} \varphi & =x_{\tau_{\alpha}(\varphi)}(; \alpha, \varphi), \varphi \neq 0 \\
A_{\alpha} 0 & =0
\end{aligned}
$$


é completamente contínua e $A_{\alpha} I_{\alpha} \subset K_{\alpha}$.

(iv) $\exists a>0$ tal que

$$
\inf \left\{\left\|\pi_{\alpha} x_{t}\right\|, x_{t}=x_{t}(. ; \alpha, \varphi), \varphi \in I_{\alpha},\|\varphi\|=a, 0 \leq t \leq \tau_{\alpha}(\varphi)\right\}>0
$$

Então 0 é ponto ejetivo de $A_{\alpha}, \quad \alpha>\alpha_{0}$.

Observemos que a hipótese ( $i)$ do Lema 2.15 é consequência de $\alpha$ pertencer a $\left(\alpha_{0}, \infty\right)$. As hipóteses (ii),(iii), (iv) seguem dos Lemas (2.6), (2.7) e (2.8), respectivamente. Sua demonstração ,que pode ser encontrada em [16], será feita no capítulo 4, num contexto mais geral.

Demonstração do Teorema 2.1: Sejam $S=K_{\alpha}$ e $A=A_{\alpha}, \alpha>\alpha_{0}$, do Lema 2.1. Seja $N>M$, onde $M$ é um limitante para a função $\mathrm{F}$ e suponhamos que $\varphi \in K_{\alpha}^{-},\|\varphi\|=N$ e $A_{\alpha} \varphi=\lambda \varphi$.

Então,

$$
x\left(\tau_{\alpha}(\varphi)+\theta ; \alpha, \varphi\right)=\lambda \varphi(\theta)
$$

para algum $\theta \in[-1,0]$ para o qual $|\varphi(\theta)|=N>M$.

Como na demonstração do Lema 2.2 , segue disto que $\lambda<1$.

O Teorema é agora, consequência imediata do Lema $2.1 . \diamond$ 


\section{Capítulo 3}

\section{Estimativas Exponenciais Uniformes}

Tratamos aqui da obtenção de estimativas exponenciais para as soluções de uma família a um parâmetro de equações diferenciais-diferença lineares, a qual inclui a linearização de $(2.1-\alpha)$ em torno da solução nula. Tais estimativas são obtidas a partir da localização das raizes características, e independem do parâmetro, quando este varia num compacto.

Quando as partes reais das raízes características são negativas e não se acumulam no zero, a propriedade acima se transfere à equasão não linear original, obtendo-se com isso estabilidade assintótica da solução nula com uma limitação inferior para a bacia de atração, independente do parâmetro restrito a compactos.

Consideremos a família de equações lineares autônomas

$$
\dot{x}(t)=\alpha A x(t)+\alpha B x(t-1)
$$

onde $x=\operatorname{col}\left(x_{1}, \ldots, x_{n}\right), \alpha$ parâmetro real positivo, A e B matrizes reais de ordem $\mathrm{n}$.

Seja $\phi=\operatorname{col}\left(\phi_{1}, \ldots, \phi_{\mathbf{n}}\right) \in \mathcal{C}\left([-1,0], \mathbf{R}^{\mathbf{n}}\right),\|\phi\|=\sup _{-1 \leq \theta \leq 0}|\phi(\theta)|$.

Dada $x_{\alpha}(t) \in \mathcal{C}\left([-1,+\infty), \mathbf{R}^{\mathrm{n}}\right)$ solução de $(3.1-\alpha),\left.x\right|_{[-1,0]}=\phi$, podemos escrevê-la na forma equivalente

$$
\left\{\begin{array}{l}
x_{\alpha}(t)=\phi(0)+\int_{0}^{t}\left[\alpha A x_{\alpha}(s)+\alpha B x_{\alpha}(s-1)\right] d s, \quad t \geq 0 \\
x_{\alpha}(t)=\phi(t), \quad-1 \leq t \leq 0
\end{array}\right.
$$

a partir da qual prova-se como em Hale $[9$, cap1,T.3.1] que

$$
\left|x_{\alpha}(t)\right| \leq a(\alpha) e^{\alpha b t}|\phi|, \quad t \geq 0
$$


$\operatorname{com} a(\alpha)=1+\alpha|B|, b=|A|+|B|$.

A norma matricial |.| indica a norma da matriz como transformação linear do $\mathbf{R}^{\mathbf{n}}$.

A equação característica de $(3.1-\alpha)$ é:

$$
\operatorname{det} H_{\alpha}(\lambda)=0, \quad H_{\alpha}(\lambda):=\lambda I-\alpha A-\alpha B e^{-\lambda}
$$

e suas soluções são as rá́zes características, também chamadas autovalores de $(3.1-\alpha)$.

Lema 3.1. Se existe uma sequência $\left(\lambda_{\jmath}\right)$ de raízes características de $(3.1-\alpha)$ tais que $\left|\lambda_{\jmath}\right| \rightarrow+\infty$, quando $\jmath \rightarrow+\infty$, então $\Re \lambda_{\jmath} \rightarrow-\infty$, quando $\jmath \rightarrow+\infty$. Assim, dado a $\in \mathbf{R}$, há no máximo um número finito de raízes caracteristicas à direita da reta $\Re \lambda=a$.

Demonstração: Seja

$$
p_{k}(s):=\prod_{i=1}^{k}\left(\alpha a_{\imath}+\alpha b_{\imath} s\right)
$$

$\operatorname{com} \alpha>0, a_{\imath}, b_{\imath} \in \mathbf{R}, s \in \mathbf{C}$. Então

$$
\begin{gathered}
\operatorname{det} H_{\alpha}(\lambda)=\lambda^{n}+p_{1}\left(e^{-\lambda}\right) \lambda^{n-1}+\ldots+p_{n-1}\left(e^{-\lambda}\right) \lambda+p_{n}\left(e^{-\lambda}\right) . \\
\operatorname{det} H_{\alpha}(\lambda)=0 \Leftrightarrow-\lambda=p_{1}\left(e^{-\lambda}\right)+\frac{p_{2}\left(e^{-\lambda}\right)}{\lambda}+\ldots \frac{p_{n}\left(e^{-\lambda}\right)}{\lambda^{n-1}}
\end{gathered}
$$

Assim

$$
|\lambda| \leq \sum_{k=1}^{n} \frac{\widetilde{p}_{k}\left(e^{-\Re \lambda}\right)}{|\lambda|^{k-1}}, \quad \tilde{p}_{k}(u):=\prod_{i=1}^{k}\left(\alpha\left|a_{\imath}\right|+\alpha\left|b_{\imath}\right| u\right), u \in \mathbf{R} .
$$

Se $|\lambda| \rightarrow+\infty$, então alguma parcela de (3.4) tende a $+\infty$, o que implica que $\Re \lambda \rightarrow$ $-\infty$.

Do que já foi provado, segue que dado $a \in \mathbf{R}, S=\left\{\lambda \mid \operatorname{det} H_{\alpha}(\lambda)=0\right.$ e $\left.\Re \lambda>a\right\}$ é limitado. Como $\operatorname{det} H_{\alpha}(\lambda)$ é uma função inteira, ela tem no máximo um número finito de zeros em qualquer compacto, de onde segue o resultado. $\diamond$ 
Lema 3.2. Seja $J_{0} \subset(0,+\infty)$ compacto e suponhamos que as raizes caracteristicas de

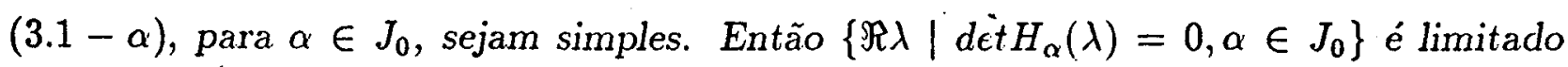
superiormente.

\section{Demonstração:}

$$
\beta_{\alpha}:=\max \left\{\Re \lambda \mid \operatorname{det} H_{\alpha}(\lambda)=0\right\}
$$

é uma função de $\alpha>0$, pelo Lemạ 3.1. Como $J_{0}$ é compacto, basta mostrar que tal função é contínua.

Sejam $\tilde{\alpha}>0$ e $b \in \mathbf{R}$ tais que

$$
F_{b}=\left\{\lambda \mid \operatorname{det} H_{\tilde{\alpha}}(\lambda)=0, \Re \lambda>b\right\} \neq 0 .
$$

Para algum $m \geq 1, F_{b}=\left\{\tilde{\lambda}_{1}, \cdots, \tilde{\lambda}_{m}\right\}$. Assim

$$
\beta_{\tilde{\alpha}}=\max \left\{\Re \tilde{\lambda}_{\jmath}, \jmath=1,2 \ldots, m\right\}
$$

Como as raizes características são simples e o campo vetorial definido em $(3.1-\alpha)$ é contínuamente diferenciável em $\alpha$, segue que $\Re \lambda_{\jmath}(\alpha), \jmath=1,2, \ldots, m$ são funções contínuas para $\alpha \in[\tilde{\alpha}-\delta, \tilde{\alpha}+\delta]$ e algum $\delta>0(\operatorname{ver}[9, \operatorname{cap} 7$, Lema 2.2$])$.

Consequentemente,

$$
\beta_{\alpha}=\max \left\{\Re \lambda_{\jmath}(\alpha), \jmath=1,2 \ldots, m\right\}
$$

e é, portanto, contínua para $\alpha \in[\tilde{\alpha}-\delta, \tilde{\alpha}+\delta]$.

Como $\tilde{\alpha}$ é arbitrário, segue o resultado. $\diamond$

Se $x_{\alpha}^{1}(t), x_{\alpha}^{2}(t), \ldots, x_{\alpha}^{n}(t)$ são soluções de $(3.1-\alpha)$ para algum $\alpha>0$, dizemos que $Y_{\alpha}(t)=\left(x_{\alpha}^{1}(t), \ldots, x_{\alpha}^{n}(t)\right)$ é uma matriz solução de $(3.1-\alpha)$.

$X_{\alpha}(t)$ com $t \geq-1$ é chamada matriz fundamental de $(3.1-\alpha)$ se é a matriz solução de $(3.1-\alpha)$ tal que $\left.X_{\alpha}(t)\right|_{[-1,0]} \equiv \Psi(t)$, onde 


$$
\Psi(t)=\left\{\begin{array}{l}
0_{n \times n}, t<0 \\
I_{n x n}, t=0
\end{array}\right.
$$

Tal definição é consistente, já que o mesmo processo recursivo usado para condições iniciais continuas garante existência e unicidade de $X_{\alpha}(t)$.

Além disso, $X_{\alpha}(t)$ é de variação limitada em qualquer conjunto compacto de $[-1, \infty)$ e vale para $\mathrm{X}_{\alpha}(t)$ a seguinte estimativa exponencial

$$
\left|X_{\alpha}(t)\right| \leq a(\alpha) e^{\alpha b t}|\Psi|, t \geq 0
$$

$\operatorname{com} a(\alpha)=1+\alpha|B|, b=|A|+|B|$.

Os lemas a seguir fazem parte da teoria de Transformadas de Laplace e não serão demonstrados.

Lema 3.3 (Existência da Transformada de Laplace). Se $F:[0, \infty) \rightarrow \mathbf{R}^{\mathbf{n}}$ é mensurável e satisfaz $|F(t)| \leq a \epsilon^{b t}, t \in[0, \infty)$, para algumas constantes a e $b$, então a Transformada de Laplace $\mathcal{L}(F)$ definida por

$$
\mathcal{L}(F)(\lambda)=\int_{0}^{\infty} e^{-\lambda t} F(t) d t
$$

existe e é uma função analítica de $\lambda$ para $\Re \lambda>b$.

Observemos que se $F:[0, \infty) \rightarrow \mathbf{R}^{\mathbf{n}}$ tem coordenadas $F_{1}, F_{2}, \ldots, F_{n}$, isto é, $F=$ $\left(F_{1} ; F_{2}, \ldots, F_{n}\right)$, então $\mathcal{L}(F)=\left(\mathcal{L}\left(F_{1}\right), \cdots, \mathcal{L}\left(F_{n}\right)\right)$.

Usaremos muitas vezes a seguinte notação

$$
\int_{(c)}=\lim _{T \rightarrow+\infty} \frac{1}{2 \pi i} \int_{c-i T}^{c+i T}
$$

onde $c$ é um número real.

Lema 3.4 (Teorema da Inversão). Sejam b uma constante positiva e $F:[0, \infty) \rightarrow \mathbf{R}^{\mathbf{n}}$ tais que $F$ é de variação limitada sôbre qualquer conjunto compacto de $[0, \infty)$ e $F(t) \epsilon^{-b t}$ é Lebesgue integrável em $[0, \infty)$. Então, para qualquer $c>b$,

$$
\int_{(c)} \mathcal{L}(F)(\lambda) e^{\lambda t} d \lambda=\left\{\begin{array}{l}
1 / 2\left[F\left(t_{+}\right)+F\left(t_{-}\right)\right], \quad t>0 \\
1 / 2 F\left(0_{+}\right), \quad t=0 .
\end{array}\right.
$$


Teorema 3.1. Para cada $\alpha>0$, existe constante $c_{\alpha}$ tal que a matriz fundamental $X_{\alpha}(t)$ de $(3.1-\alpha)$ satisfaz

$$
\mathcal{L}\left(X_{\alpha}\right)(\lambda)=H_{\alpha}^{-1}(\lambda)
$$

para todo $\lambda \in \mathbf{C}$ tal que $\Re \lambda>c_{\alpha}$.

Além disso, para qualquer constante $c>c_{\alpha}$,

$$
X_{\alpha}(t)=\int_{(c)} H_{\alpha}^{-1}(\lambda) e^{\lambda t} d \lambda, t>0
$$

Demonstração: Desde que para todo $\alpha>0, X_{\alpha}(t)$ é mensurável e satisfaz (3.6), segue do Lema 3.3 que

$$
\mathcal{L}\left(X_{\alpha}\right)(\lambda)=\int_{0}^{\infty} e^{-\lambda t} X_{\alpha}(t) d t
$$

é uma função analítica de $\lambda$ para $\Re \lambda>\alpha b$.

Da equação $(3.1-\alpha)$

$$
\int_{0}^{\infty} e^{-\lambda s} \dot{X}_{\alpha}(s) d s=\alpha A \int_{0}^{\infty} e^{-\lambda s} X_{\alpha}(s) d s+\alpha B \int_{0}^{\infty} e^{-\lambda s} X_{\alpha}(s-1) d s
$$

e então, por uma integração por partes,

$$
-I+\lambda \mathcal{L}\left(X_{\alpha}\right)(\lambda)=\alpha A \mathcal{L}\left(X_{\alpha}\right)(\lambda)+\alpha \epsilon^{-\lambda} B \mathcal{L}\left(X_{\alpha}\right)(\lambda)
$$

ou seja,

$$
H_{\alpha}(\lambda) \mathcal{L}\left(X_{\alpha}\right)(\lambda)=I
$$

para $\alpha>0, \Re \lambda>\alpha b$.

Sabemos que $H_{\alpha}(\lambda)$ é inversivel para todo $\lambda$ tal que $\Re \lambda>\beta_{\alpha}, \operatorname{com} \beta_{\alpha}$ dado em (3.5). Assim,

$$
H_{\alpha}^{-1}(\lambda)=\mathcal{L}\left(X_{\alpha}\right)(\lambda)
$$

para todo $\lambda$ tal que $\Re \lambda>c_{\alpha}$, onde $c_{\alpha}=\max \left\{a b, \beta_{\alpha}\right\}$. 
Estamos agora em condições de aplicar o Lema 3.4 à função $X_{\alpha}(t)$ com constante c maior que $c_{\alpha}$, já que

$$
\left|X_{\alpha}(t) e^{-c_{\alpha} t}\right| \leq a(\alpha) e^{\alpha b t} e^{-c_{\alpha} t} \leq a(\alpha), \quad t \geq 0
$$

e portanto $X_{\alpha}(t) e^{-c_{\alpha} t}$ é Lebesgue integrável em $[0, \infty)$, o que finaliza a demonstração. $\diamond$

Corolário 3.1. Seja $J_{0}$ um subconjunto limitado de $(0, \infty)$ tal que $\left\{\Re \lambda \mid \operatorname{det} H_{\alpha}(\lambda)=\right.$ $\left.0, \alpha \in J_{0}\right\}$ é limitado superiormente. Então existe constante $\bar{c}$ tal que para todo $\alpha \in J_{0}$,

$$
\mathcal{L}\left(X_{\alpha}\right)(\lambda)=H_{\alpha}^{-1}(\lambda), \quad \lambda \in \mathbf{C} \in \Re \lambda>\bar{c}
$$

e

$$
X_{\alpha}(t)=\int_{(c)} H_{\alpha}^{-1}(\lambda) e^{\lambda t} d \lambda, \quad t>0
$$

onde $c$ é um número real qualquer maior que $\bar{c}$.

Demonstração: Sejam

$$
\gamma_{0}:=\sup \left\{\Re \lambda \mid \operatorname{det} H_{\alpha}(\lambda)=0, \alpha \in J_{0} .\right\}
$$

e $\bar{\alpha}=\sup J_{0}$.

A desigualdade (3.6) implica que existem constantes positivas $a$ e $d$ tais que

$$
\left|X_{\alpha}(t)\right| \leq a \epsilon^{d t}, \quad t \geq 0, \alpha \in J_{0}
$$

De fato, basta tomar $a=1+\bar{\alpha}|B|, d=\bar{\alpha}(|A|+|B|)$.

Seja $\bar{c}=\max \left\{\gamma_{0}, d\right\}$.

Como $\bar{c} \geq c_{\alpha}$ para todo $\alpha \in J_{0}$, segue o resultado. $\diamond$

Corolário 3.2. Se $J_{0} \subset(0, \infty)$ é compacto e as raizes características de $(3.1-\alpha)$ para $\alpha \in J_{0}$ são simples, valem as mesmas conclusões de corolário 3.1 .

Demonstração: segue trivialmente do Lema 3.2 e corolário $3.1 . \diamond$ 
Teorema 3.2. Sejam $J_{0}$ um subconjunto limitado de $(0, \infty)$ e $\gamma_{0}<\infty$, com $\gamma_{0}$ dado em (3.7). Então para qualquer $\gamma>\gamma_{0}, \exists K=K(\gamma)>0$ tal que a matriz fundamental de $(3.1-\alpha), X_{\alpha}(t)$, satisfaz

$$
\left|X_{\alpha}(t)\right| \leq I e^{\gamma t}, t \geq 0, \alpha \in J_{0}
$$

Demonstração: Provemos primeiramente que para $\alpha \in J_{0}$,

$$
X_{\alpha}(t)=\int_{(\gamma)} H_{\alpha}^{-1}(\lambda) e^{\lambda t} d \lambda, \quad t>0
$$

onde $\gamma$ é uma constante qualquer maior que $\gamma_{0}$.

Para mostrar isso, vamos considerar a integração da função $H_{\alpha}^{-1}(\lambda) e^{\lambda t}$ no plano complexo ao longo da fronteira do retângulo B de lados $L_{1} M_{1} L_{2} M_{2}$ (ver fig.3.1) na direção indicada, onde

$$
\begin{array}{ll}
L_{1}=\{c+i \tau \mid-T \leq \tau \leq T\}, & M_{1}=\{\sigma+i T \mid \gamma \leq \sigma \leq c\} \\
L_{2}=\{\gamma+i \tau \mid-T \leq \tau \leq T\}, & M_{2}=\{\sigma-i T \mid \gamma \leq \sigma \leq c\}
\end{array}
$$

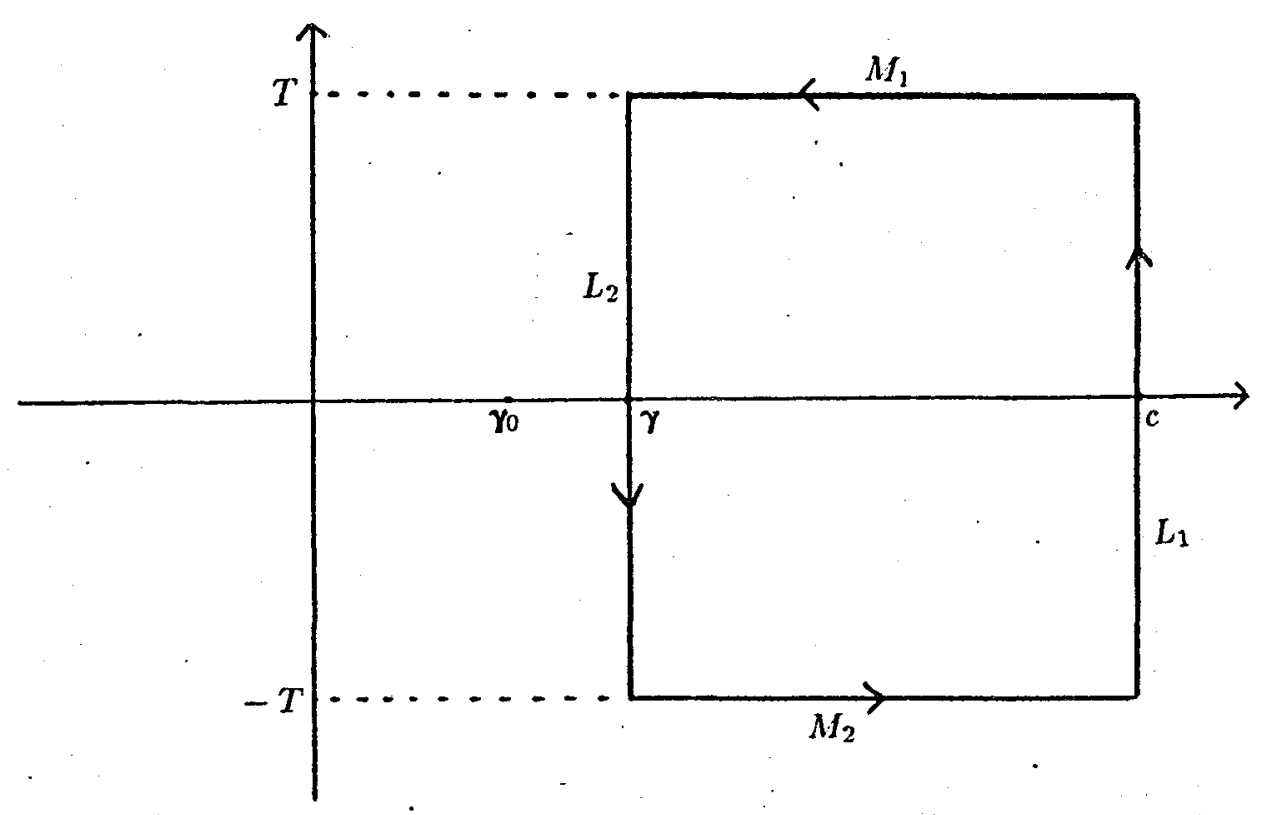


Temos que $H_{\alpha}(\lambda)$ é inversivel e $H_{\alpha}^{-1}(\lambda) \epsilon^{\lambda t}$ é analitica, para todo $\alpha \in J_{0}$ e todo $\lambda$ tal que $\Re \lambda>\gamma_{0} ;$ assim

$$
\int_{B} H_{\alpha}^{-1}(\lambda) \epsilon^{\lambda t} d \lambda=0
$$

Para prorarmos (3.9), basta mostrarmos que

$$
\int_{M_{1}} H_{\alpha}^{-1}(\lambda) e^{\lambda t} d \lambda \text { e } \int_{M_{2}} H_{\alpha}^{-1}(\lambda) e^{\lambda t} d \lambda
$$

tendem a zero quando $T \rightarrow+\infty$.

De $(3.3-a)$ segue que

$$
I=\lambda H_{\alpha}^{-1}(\lambda)-\alpha A H_{\alpha}^{-1}(\lambda)-\alpha \epsilon^{-\lambda} B H_{\alpha}^{-1}(\lambda)
$$

e então

$$
\left(|\lambda|-\alpha|A|-\alpha e^{-\Re \lambda}|B|\right)\left|H_{\alpha}^{-1}(\lambda)\right| \leq 1
$$

para todo $\lambda \operatorname{com} \Re \lambda>\gamma_{0}, \alpha \in J_{0}$.

Para $\lambda \in M_{1}, \alpha \in J_{0}$,

$|\lambda|-\alpha|A|-\alpha e^{-\Re \lambda}|B|=\left(\sigma^{2}+T^{2}\right)^{1 / 2}-\alpha\left(|A|+e^{-\sigma}|B|\right) \geq\left(\sigma^{2}+T^{2}\right)^{1 / 2}-\bar{\alpha}\left(|A|+\epsilon^{-\gamma}|B|\right)$

$\operatorname{com} \bar{\alpha}=\sup J_{0}$. Então existe $T_{0} \geq 0$, independente de $\alpha$, tal que para $T \geq T_{0}$,

$$
|\lambda|-\alpha|A|-\alpha e^{-\Re \lambda}|B|>\frac{T}{2}
$$

para $\alpha \in J_{0}, \lambda \in M_{1}$.

Em consequência de (3.10) e (3.11),

$$
\left|\int_{M_{1}} H_{\alpha}^{-1}(\lambda) e^{\lambda t} d \lambda\right| \leq \frac{2}{T} e^{c t}(c-\gamma) \rightarrow 0, \text { quando } T \rightarrow+\infty .
$$

Analogamente, mostra-se que 


$$
\int_{M_{2}} H_{\alpha}^{-1}(\lambda) e^{\lambda t} d \lambda \rightarrow 0, \text { quando } T \rightarrow+\infty .
$$

Sejam $\dot{\lambda}=\gamma+i \tau$ e $\alpha \in J_{0}$. Definimos

$$
G_{\alpha}(\lambda)=H_{\alpha}^{-1}(\lambda)-\left(\lambda-\gamma_{0}\right)^{-1} I=\left(\lambda-\gamma_{0}\right)^{-1}\left[\left(\lambda-\gamma_{0}\right) I-H_{\alpha}(\lambda)\right] \dot{H}_{\alpha}^{-1}(\lambda)
$$

Formalmente, tomando $T_{0}$ como acima,

$$
\begin{aligned}
\int_{(\gamma)}\left|G_{\alpha}(\lambda)\right| d \lambda= & \lim _{T \rightarrow+\infty} \frac{1}{2 \pi}\left[\int_{-T}^{-T_{0}}\left|G_{\alpha}(\gamma+i \tau)\right| d \tau+\int_{-T_{0}}^{T_{0}}\left|G_{\alpha}(\gamma+i \tau)\right| d \tau+\right. \\
& \left.+\int_{T_{0}}^{T}\left|G_{\alpha}(\gamma+i \tau)\right| d \tau\right]
\end{aligned}
$$

Da analiticidade de $G_{\alpha}(\lambda)$ em $L_{2}$ e continuidade em $\alpha$ segue que $\exists K_{1}>0$ tal que

$$
\int_{-T_{0}}^{T_{0}}\left|G_{\alpha}(\gamma+i \tau)\right| d \tau \leq 2 I_{1} T_{0}, \quad \forall \alpha \in J_{0}
$$

Além disso, para $|\tau| \geq T_{0}, \alpha \in J_{0}$, segue de (3.10),(3.11) e (3.12),

$$
\left|G_{\alpha}(\gamma+i \tau)\right| \leq \frac{2}{\tau^{2}}\left(\left|\gamma_{0}\right|+\bar{\alpha}|A|+\bar{\alpha}|B| \epsilon^{-\gamma}\right)
$$

Assim, por (3.13), (3.14), (3.15)

$$
\left|\int_{(\gamma)}\right| G_{\alpha}(\lambda)|d \lambda| \leq I_{2}
$$

para todo $\alpha \in J_{0}$, com

$$
K_{2}=\frac{1}{2 \pi}\left[2 K_{1} T_{0}+\frac{4}{T_{0}}\left(\left|\gamma_{0}\right|+\bar{\alpha}|A|+\bar{\alpha}|B| e^{-\gamma}\right)\right]
$$

independente de $\alpha$.

Consequentemente

$$
\left|\int_{(\gamma)} G_{\alpha}(\lambda) \epsilon^{\lambda t} d \lambda\right| \leq I_{2} e^{\gamma t}
$$


Além disso, por processo standard da teoria de funções complexas, mostra-se que

$$
\left|\int_{(\gamma)}\left(\lambda-\gamma_{0}\right)^{-1} e^{\lambda t} d \lambda\right| \leq 4 \pi e^{\gamma t}
$$

De (3.12), (3.16) e (3.17) segue que

$$
\left|X_{\alpha}(t)\right|=\left|\int_{(\gamma)} H_{\alpha}^{-1}(\lambda) e^{\lambda t} d \lambda\right| \leq I \dot{\gamma} e^{\gamma t}
$$

para $t \geq 0, \alpha \in J_{0}$, para alguma constante $\Pi=K(\gamma)$ independente de $\alpha . \diamond$

A soluçào $x_{\alpha}(\phi)(t)$ da equação $(3.1-\alpha), \alpha>0$, que coincide com $\phi$ em $[-1,0]$ pode ser representada por

$$
x_{\alpha}(\phi)(t)=X_{\alpha}(t) \phi(0)+B \int_{-1}^{0} X_{\alpha}(t-\theta-1) \phi(\theta) d \theta, \quad t \geq 0
$$

$(\operatorname{ver}[9, \operatorname{cap} 1$, Teo 6.1$])$.

Teorema 3.3. Sob as hipóteses do Teorema 3.2, para qualquer $\gamma>\gamma_{0}$, existe constante $K=K(\gamma)>0$, independente de $\alpha$, tal que a solução $x_{\alpha}(\phi)(t)$ da equação $(3.1-\alpha), \alpha \in J_{0}$, com condição inicial $\phi$ em $[-1,0]$ satisfaz

$$
\left|x_{\alpha}(\phi)(t)\right| \leq \pi e^{\gamma t}|\phi|, \quad \text { para } t \geq 0
$$

Em particular, se $\gamma_{0}<0$, podemos escolher $\gamma_{0}<\gamma<0$ e concluir que a origem é globalmente exponencialmente estável.

Demonstração: Segue naturalmente da limitação exponencial de $X_{\alpha}(t)$ dada no Teorema 3.2 e de (3.18). $\diamond$

É sabido que para as nossas equações vale um teorema correspondente ao Teorema da Primeira Aproximação de Poincaré-Liapunov. Assim, o decaimento exponencial expresso em (3.19) se transfere, embora não globalmente, a uma família de equações perturbadas não lineares da forma. 


$$
\dot{x}(t)=\alpha A x(t)+\alpha B x(t-1)+\alpha \dot{f}(x(t), x(t-1))
$$

onde $x=\operatorname{col}\left(x_{1}, \ldots, x_{n}\right), \quad \alpha$ parâmetro real positivo, $\mathrm{A}$ e B matrizes reais de ordem $\mathrm{n} \mathrm{e}$ $f: \mathbf{R}^{\mathbf{n}} \times \mathbf{R}^{\mathbf{n}} \rightarrow \mathbf{R}^{\mathrm{n}}$ contínua, diferenciável na origem, $f(0)=0$ e $f^{\prime}(0)=0$.

Demonstraremos a seguir que, embora se perca o caráter global da estabilidade ao passar da equação linear para $(3.20-\alpha)$, obtemos uma estimativa uniforme em $\alpha$ para a bacia de atração da solução nula.

Denotemos por $x_{\alpha}(\phi, f)(t)$ a solução de $(3.20-\alpha)$, definida em seu intervalo maximal, a qual coincide com $\phi$ em $[-1,0]$.

Teorema 3.4. Sejam as hipóteses do Teorema $3.2 \mathrm{com} \gamma_{0}<0$. Então existem constantes positivas $\delta, L$ e $\eta$ tais que $\left|x_{\alpha}(\phi, f)(t)\right| \leq L e^{-\eta t}\|\phi\|$, para todo $t \geq 0, \alpha \in J_{0}$, e $\|\phi\|<\delta$.

Demonstração: Seja $\left[-1, b_{+}\right) \circ$ intervalo maximal de existência da solução $x_{\alpha}(\phi, f)(t)$.

A Fórmula da Variação das Constantes para $(3.20-\alpha)$ é

$$
x_{\alpha}(\phi, f)(t)=x_{\alpha}(\phi)(t)+\alpha \int_{0}^{t} X_{\alpha}(t-s) f(x(s), x(s-1)) d s
$$

para $0 \leq t \leq b_{+}$, onde $x_{\alpha}(\phi)(t)$ é solução de $(3.1-\alpha)$ a qual coincide com $\phi$ em $[-1,0] \mathrm{e}$ $X_{\alpha}(t)$ é a matriz fundamental de $(3.1-\alpha)$ (ver [9, cap.1, Teo 6.1]).

Segue das desigualdades (3.8)e(3.19), para $\gamma_{0}<\gamma<0$ e $\bar{\alpha}=\sup J_{0}$,

$$
\left|x_{\alpha}(\phi, f)(t)\right| \leq K e^{\gamma t}\|\phi\|+\bar{\alpha} \Pi \int_{0}^{t} e^{\gamma(t-s)} \mid f(x(s), x(s-1) \mid d s .
$$

Da hipótese sobre a função $\mathrm{f}$, dado $0<\varepsilon_{0}<\frac{-\gamma}{\bar{\alpha} K\left[1+\epsilon^{-\gamma}\right]}, \exists \delta_{0}>0$, tal que

$$
|f(u, v)| \leq \varepsilon_{0}(|u|+|v|), \quad \text { se }|u|+|v| \leq \delta_{0} .
$$

Seja

$$
\|\phi\|<\delta:=\min \left\{\frac{\delta_{0}}{2}, \frac{\delta_{0}}{2 \pi e^{\bar{\alpha} K \varepsilon_{0}\left[1+e^{-\gamma}\right]}}\right\}
$$


e suponhamos que exista $t_{0} \in\left(0, b_{+}\right)$tal que,

$$
\left|x_{\alpha}(\phi, f)(t)\right|<\delta_{0}, \quad \text { se } 0 \leq t<t_{0} \quad \text { e } \quad\left|x_{\alpha}(\phi, f)\left(t_{0}\right)\right|=\delta_{0}
$$

Para $0 \leq t \leq t_{0}$, segue de (3.22) que

$$
e^{-\gamma t}\left|x_{\alpha}(\phi, f)(t)\right| \leq \Pi\|\phi\|+\bar{\alpha} I \varepsilon_{0} \int_{0}^{t} e^{-\gamma s}|x(s)| d s+\bar{\alpha} \Lambda_{\varepsilon_{0}} \int_{0}^{t} e^{-\gamma s}|x(s-1)| d s,
$$

e então,

$$
e^{-\gamma t}\left|x_{\alpha}(\phi, f)(t)\right| \leq K\|\phi\|+\bar{\alpha} I_{\varepsilon} \varepsilon_{0}\left[1+e^{-\gamma}\right] \int_{-1}^{t} e^{-\gamma s}|x(s)| d s
$$

Segue desta desigualdade (ver [9, cap.1, Lema 3.1]), que

$$
e^{-\gamma t}\left|x_{\alpha}(\phi, f)(t)\right| \leq K e^{\bar{\alpha} K \varepsilon_{0}\left[1+e^{-\gamma}\right](t+1)}\|\phi\|
$$

e assim

$$
\left|x_{\alpha}(\phi, f)(t)\right| \leq L \epsilon^{-\eta t}\|\phi\|, \quad 0 \leq t \leq t_{0}
$$

onde $L=K e^{\bar{\alpha} K \varepsilon_{0}\left[1+e^{-\gamma}\right]}$ e $\eta=-\gamma-\bar{\alpha} K \varepsilon_{0}\left[1+e^{-\gamma}\right]$.

Segue de (3.23) e da definição de $\delta$,

$$
\left|x_{\alpha}(\phi, f)(t)\right| \leq \frac{\delta_{0}}{2}, \quad \text { para } \quad 0 \leq t \leq t_{0}
$$

o que contraria a definição de $t_{0}$.

Isto implica que

$$
\left|x_{\alpha}(\phi, f)(t)\right|<\delta_{0}, \quad \forall t \in\left[0, b_{+}\right) .
$$

Consequentemente, $b_{+}=+\infty$ e vale (3.23) para todo $t \geq 0 . \diamond$

A família de equações lineares no $\mathbf{R}^{2}$ 


$$
\begin{gathered}
\dot{x}(t)=-\alpha x(t)+\alpha B x(t-\ddot{1}) \\
B=\left(\begin{array}{cc}
0 & \delta_{1} \\
\delta_{2} & 0
\end{array}\right), \quad \delta_{1}>0, \quad \delta_{2}<0, \quad \delta=\sqrt{-\delta_{1} \delta_{2}}>1, \quad \alpha>0 .
\end{gathered}
$$

constituem linearizações em torno da origem das equações $(2.1-\alpha)$. Como consequência, temos o seguinte resultado.

Corolário 3.3. Seja $J_{0}$ um compacto de $\left(0, \alpha_{0}\right)$, com $\alpha_{0}$ definido em (1.7). Então existem constantes positivas $\delta, L$ e $\eta$ tais que se $\alpha \in J_{0},\|\varphi\|<\delta$, a solução $x(; \alpha, \varphi)$ de $(2.1-\alpha)$ satisfaz

$$
|x(t ; \alpha, \varphi)| \leq L e^{-\eta t}\|\varphi\|, \quad t \geq 0
$$

Demonstração: Para cada $\alpha \in J_{0}$, seja $\lambda_{0}(\alpha)$ a raiz característica da equação $(3.24-\alpha)$ pertencente a $S_{0}$.

Temos que $\Re \lambda_{0}(\alpha)<0$ e, pelo Lema 1.6 ,

$$
\beta_{\alpha}:=\max \left\{\Re \lambda \mid \operatorname{det} H_{\alpha}(\lambda)=0\right\}=\Re \lambda_{0}(\alpha)
$$

Além disso, $\lambda_{0}(\alpha)$ é uma função contínua de $\alpha$ e então $\beta_{\alpha} \leq \gamma_{0}, \forall \alpha \in J_{0}$, para algum $\gamma_{0}<0$

O resultado segue, agora, por aplicação imediata do Teorema 3.4 com

$$
f(x(t), x(t-1))=F(x(t-1))-B x(t-1) .
$$




\section{Capítulo 4}

\section{Continuação global}

Este capítulo final foi motivado pela existência de soluções periódicas com período $T>4$ para a equação $(2.1-\alpha), \alpha>\alpha_{0}$, conforme Teorema 2.1. O Teorema 1.1 sugere que essas soluções devem constituir uma extensão do ramo da bifurcação local de Hopf emanante de $\alpha=\alpha_{0}$, uma vez que este consiste de soluções com período próximo de $\frac{2 \pi}{b_{0}}>4$.

Sob hipóteses bastante gerais os resultados seguintes confirmam essa conjectura. Foram inspirados no problema escalar estudado por Mallet-Paret e Nussbaum em [12], embora não se trate de uma simples adaptação. As técnicas aqui utilizadas são basicamente distintas, uma vez que o problema no plano apresenta dificuldades próprias.

Faremos uma aplicação de um teorema de Nussbaum, enunciado em [9,cap 11,Teo 3.1] e provado, num contexto mais geral, em [13]. Tal teorema estabelece a existência de um ramo ilimitado de soluções periódicas a partir de uma bifurcação de Hopf. Para enunciá-lo, coloquemos antes a definição de ponto atrativo de um operador, em contraposição à de ponto ejetivo-definição 2.1 .

Definição 4.1: Sejam $\mathrm{X}$ um espaço topológico, $x_{0} \in X$, U uma vizinhança aberta de $x_{0}$ em $\mathrm{X}$ e $A: U \rightarrow X$ contínua. O ponto $x_{0}$ é chamado ponto atrativo de $\mathrm{A}$ se existir uma vizinhánça aberta $U_{0}$ de $x_{0}$, e para qualquer vizinhança aberta $\mathrm{V}$ de $x_{0}$, existir um inteiro $n=n(V)$ tal que $A^{J}\left(U_{0}\right) \subset U$ para $0 \leq \jmath \leq n$ e $A^{\jmath}\left(U_{0}\right) \subset V$ para $\jmath \geq n$.

Teorema 4.1. Seja $S$ um subconjunto fechado e convexo de um espaço de Banach $Y, 0$ um ponto extremo de $S, S \neq\{0\}$ e $J=(a, \infty), \quad a \geq-\infty$. Suponhamos $F: S \times J \rightarrow S$ satisfazendo as seguintes hipóteses: 
(i) $F(0, \alpha)=0$ para $\alpha \in J$.

(ii) F é completamente contínua.

(iii) Existe $\alpha_{0} \in J$ tal que, para qualquer intervalo compacto $J_{0} \subset J-\left\{\alpha_{0}\right\}$, existe um $\varepsilon=\varepsilon\left(J_{0}\right)>0$ tal que $F(x, \alpha) \neq x$ para $\alpha \in J_{0}, \quad 0<|x| \leq \varepsilon$.

(iv) Existe um intervalo aberto $I_{0}$ contendo $\alpha_{0}$ tal que 0 é um ponto atrativo de $F(., \alpha)$ para $\alpha \in I_{0}, \quad \alpha<\alpha_{0}$, e 0 é um ponto ejetivo de $F(., \alpha)$ para $\alpha \in I_{0}, \quad \alpha>\alpha_{0}$, ou vice-versa.

(v) Existe um $\alpha_{1} \in J$ tal que $F(x, \alpha)=x$ para $x \neq 0$ implica $\alpha \geq \alpha_{1}$.

Definamos o conjunto $\mathrm{E}$ por

$$
E=\{(x, \alpha) \in S \times J \mid x \neq 0 \in F(x, \alpha)=x\}
$$

e seja $E_{0}$ a componente conexa de $\bar{E}$ que contem $\left(0, \alpha_{0}\right)$. Então $E_{0}$ é não vazia e ilimitada.

Consideremos a família de equações $(2.1-\alpha)$ e suponhamos que $F$ satisfaça, além das hipóteses (H.1) e (H.2), as seguintes:

$$
x \cdot F(x)<|x|^{2} \quad \text { se } \quad x \in \bar{B}_{M}-\{0\}
$$

onde"." denota o produto escalar usual do $\mathbf{R}^{2}$.

$$
\left|\delta_{1}+\delta_{2}\right|<2
$$

Podemos dizer que (H.3) não chega a ser uma restrição muito forte na presença de (H.1) e (H.2). Observemos 'que $F$ leva um vetor não nulo de $Q_{1}: x_{1}, x_{2} \geq 0$ em $Q_{2}: x_{2} \leq$ $0 \leq x_{1}$ e, dadas as simetrias de $F$, basta analisar o seu efeito em $Q_{1}$. - veja Figura 4.1 . 
(H.3) impõe que $\mathrm{F}(\mathrm{x})$ não pertença à regiào sombreada.

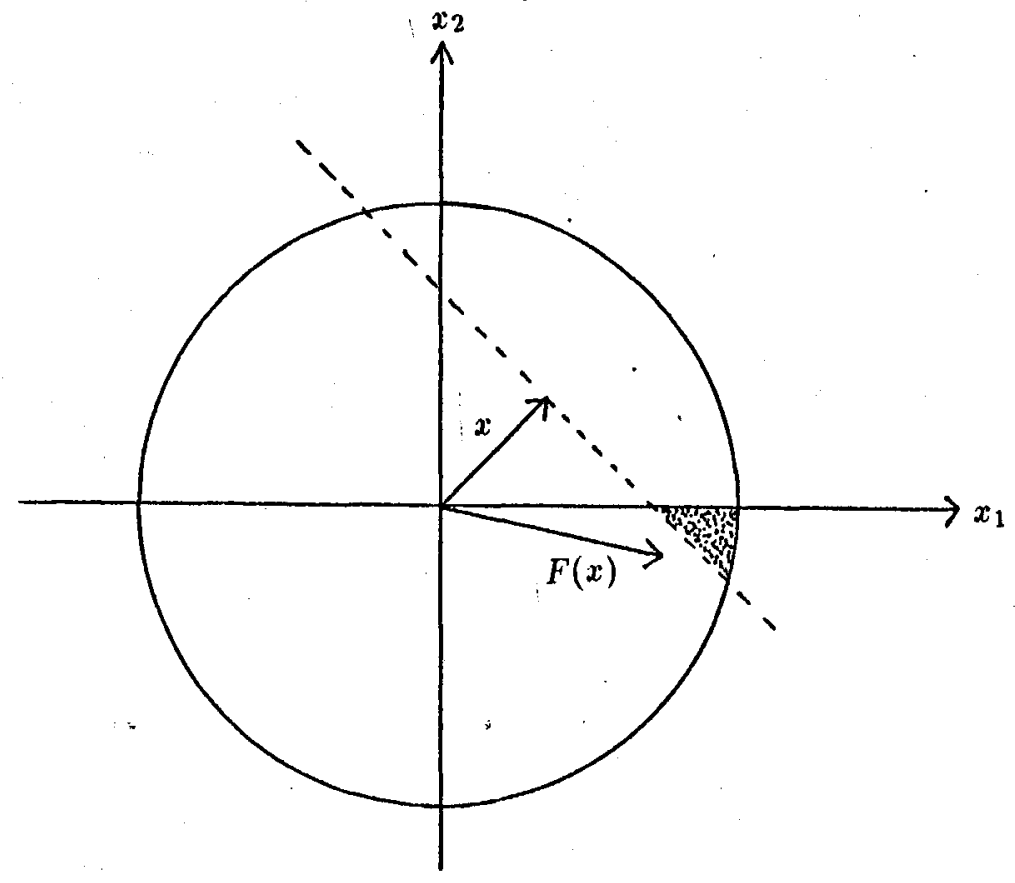

Figura 4.1

É fácil ver que (H.4) implica (H.3) numa vizinhança da origem.

Além disso, através do desenvolvimento de Taylor de $F(x)$ até a ordem 2, (H.3) resulta em

$$
\begin{gathered}
{\left[A x_{1}+B x_{2}\right] x_{1} x_{2}+O\left(|x|^{4}\right)<x_{1}^{2}+x_{2}^{2}-\left(\delta_{1}+\delta_{2}\right) x_{1} x_{2}} \\
A=\frac{1}{2} \frac{\partial^{2} F_{2}}{\partial x_{1}^{2}}(0)+\frac{\partial^{2} F_{1}}{\partial x_{1} \partial x_{2}}(0) \\
B=\frac{1}{2} \frac{\partial^{2} F_{1}}{\partial x_{2}^{2}}(0)+\frac{\partial^{2} F_{2}}{\partial x_{1} \partial x_{2}}(0)
\end{gathered}
$$

e podemos constatar, por essa desigualdade, que $\left|\delta_{1}+\delta_{2}\right| \leq 2$, e uma condição suficiente para que $\left|\delta_{1}+\delta_{2}\right|<2$ é que $A \neq \pm B$.

Um exemplo de uma função $F: \mathbf{R}^{2} \rightarrow \mathbf{R}^{2}$ satisfazendo (H.1) - (H.4) é

$$
F\left(x_{1}, x_{2}\right)=\left(2 \operatorname{arctg} x_{2},-2 \operatorname{arctg} x_{1}\right)
$$


Definição 4.2: Uma solução $x(t)$ de $(2.1-\alpha)$ é chamada solução periódica lentamente espiralante se $\exists q_{1}, q_{2}, q_{3}, q_{4}>0, q_{\jmath}>q_{\jmath-1}+1$ para $j=2,3,4$, tais que

(i) $x_{1}(-1)=0, \quad x_{1}(t)>0$ se $-1<t<q_{2}$ e $x_{1}(t)<0$ se $q_{2}<t<q_{4}$.

(ii) $x_{2}(t)>0$ se $-1<t<q_{1}$ e se $q_{3}<t<q_{4}, \quad x_{2}(t)<0$ se $q_{1}<t<q_{3}$.

(iii) $x(t)^{\circ}=x\left(t+q_{4}+1\right), \quad \forall t$.

A figura a seguir ilustra uma solução periódica lentamente espiralante.

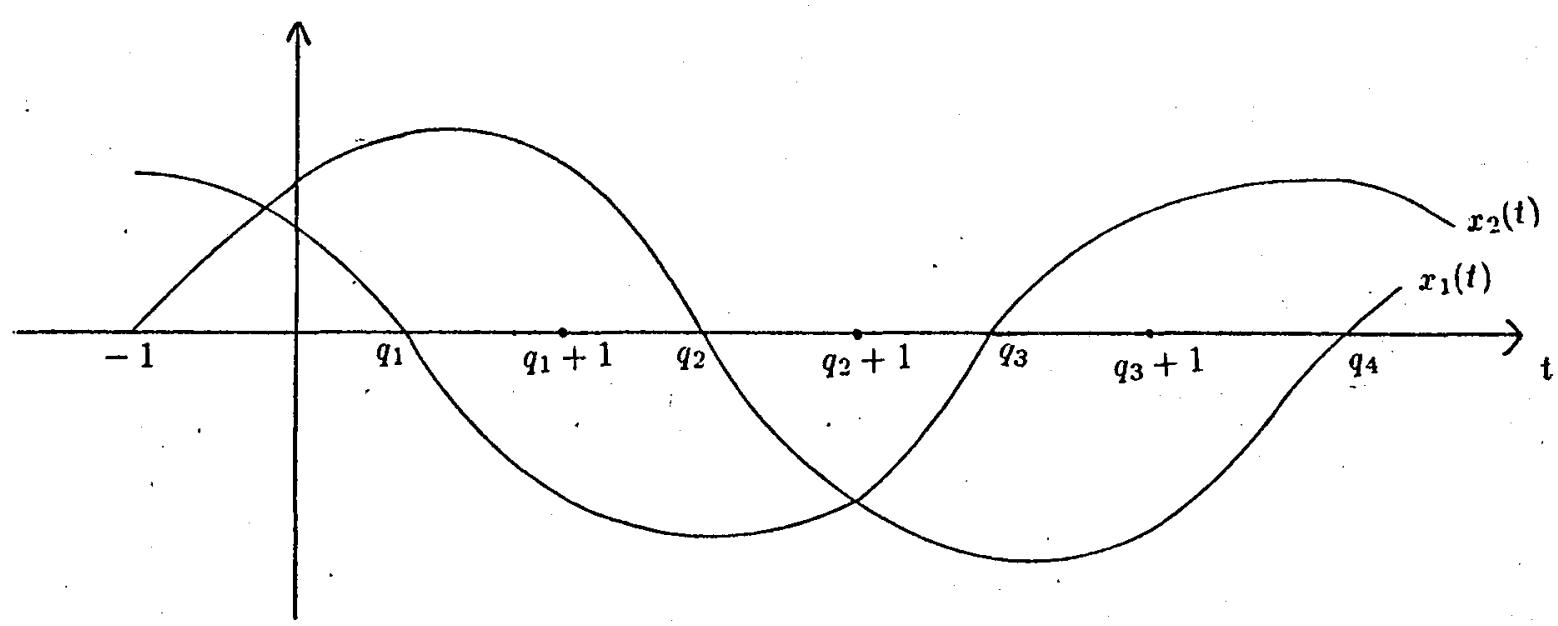

\section{Figura 4.2}

O termo lentamente espiralante é sugerido pelo fato de que a órbita envolve a origem, cruzando os semi-eixos coordenados do plano fase $x_{1} x_{2}$ em intervalos de tempo maiores que o retardamento. Essa nomenclatura é inspirada na expressão lentamente oscilante usada para equações escalares.

O período de uma solução periódica lentamente espiralante é sempre maior que quatro.

Dáda uma solução periódica lentamente espiralante de $(2.1-\alpha)$ com período $\bar{q}$, segue da definição 4.2 que

$$
\varphi:=x_{0}(\cdot) \in K-\{0\}
$$


com $\mathrm{K}$ definido em (2.3).

Além disso, da equação $(2.1-\alpha)$,

$$
\frac{d\left(e^{\alpha t} x(t)\right)}{d t}=\alpha e^{\alpha t} F(x(t-1))=\alpha e^{\alpha t} F(x(t+\bar{q}-1))
$$

e então, pela hipótese (H.1), $e^{\alpha t} \varphi_{\jmath}(t)$ é monótona crescente em $[-1,0], \jmath=1,2$.

Assim, $\varphi \in K_{\alpha},\left(K_{\alpha}\right.$ como em 2.4), e é úm ponto fixo de

$$
\begin{aligned}
A_{\alpha}: K_{\alpha} & \longrightarrow K_{\alpha} \\
A_{\alpha}(\varphi) & =x_{\tau_{\alpha}(\varphi)}(; \alpha, \varphi), \quad \varphi \neq 0 \\
A_{\alpha}(0) & =0
\end{aligned}
$$

$\operatorname{com} \tau_{\alpha}(\varphi)$ definida em $(2.10)$.

Reciprocamente, se $A_{\alpha} \varphi=\varphi$ e $\varphi \neq 0$, então $x(\cdot ; \alpha, \varphi)$ é solução periódica lentamente espiralante, em consequência da definição de $A_{\alpha}$ e resultados do capítulo 2 .

Seja $\Gamma \subset \mathbf{R} \times \mathcal{C}$ definido por

$$
\Gamma=\left\{(\alpha, \varphi) \in(0, \infty) \times \Pi \mid \varphi \in K_{\alpha}\right\}
$$

Proposição 4.1. A função

$$
\begin{array}{cl}
\tau: \Gamma-\{(\alpha, 0) \mid \alpha \in(0, \infty)\}, & \rightarrow[4, \infty) \\
\tau(\alpha, \varphi) & =\tau_{\alpha}(\varphi)
\end{array}
$$

está definida sobre um aberto de $\Gamma$ e é continua.

Demonstração: $K_{\alpha_{2}} \subset K_{\alpha_{1}}$ se $\alpha_{2}<\alpha_{1}$.

Então a desigualdade

$$
\left|\tau\left(\alpha_{1}, \varphi_{1}\right)-\tau\left(\alpha_{2}, \varphi_{2}\right)\right| \leq\left|\tau_{\alpha_{1}}\left(\varphi_{1}\right)-\tau_{\alpha_{1}}\left(\varphi_{2}\right)\right|+\left|\tau_{\alpha_{1}}\left(\varphi_{2}\right)-\tau_{\alpha_{2}}\left(\varphi_{2}\right)\right|
$$

juntamente com as continuidades de $\tau_{\alpha}$ e da solução $x(\cdot ; \alpha, \varphi)$ em relação ao parâmetro $\alpha$ garantem a continuidade da função $\tau . \diamond$

Definimos 


$$
\begin{array}{cl}
A: \Gamma & \rightarrow K \\
A(\alpha, \varphi) & =A_{\alpha}(\varphi)
\end{array}
$$

Proposição 4.2. A é completamente contínua.

Demonstração: As continuidades de $A_{\alpha}$, da função $\tau$ e das solução de $(2.1-\alpha) \mathrm{em}$ relação ao parâmetro $\alpha$, juntamente com a desigualdade

$$
\left|A\left(\alpha_{1}, \varphi_{1}\right)-A\left(\alpha_{2}, \varphi_{2}\right)\right| \leq\left|A_{\alpha_{1}}\left(\varphi_{1}\right)-A_{\alpha_{1}}\left(\varphi_{2}\right)\right|+\left|x_{\tau\left(\alpha_{1}, \varphi_{2}\right)}\left(\cdot ; \alpha_{1}, \varphi_{2}\right)-x_{\tau\left(\alpha_{2}, \varphi_{2}\right)}\left(\cdot ; \alpha_{2}, \varphi_{2}\right)\right|
$$

implicam que $A$ é contínua em $\Gamma-\{(\alpha, 0) \mid \alpha>0\}$.

Nos pontos $(\alpha, 0), \alpha>0$, a continuidade de $A$ segue do Lema 2.6.

Seja $F=\{(\alpha, \varphi) \in \Gamma \mid a \leq \alpha \leq b,\|\varphi\| \leq c\}$.

Então,

$$
A(F)=\left\{x_{\tau(\alpha, \varphi)}(\cdot ; \alpha, \varphi) \mid(\alpha, \varphi) \in F, \varphi \neq 0\right\} \cup\{0\} .
$$

Os mesmos argumentos da prova do Lema 2.2 implicam que $A(F)$ é limitado por $\max \{M, c\}$.

A equicontinuidade de $\dot{A}(\mathrm{~F})$ segue agora da continuidade da função

$$
\begin{array}{cl}
H:(0, \infty) \times \mathbf{R}^{2} \times \mathbf{R}^{2} & \rightarrow \mathbf{R}^{2} \\
H(\alpha, u, v) & =-\alpha u+\alpha F(v)
\end{array}
$$

Vimos que sob as hipóteses (H.1) e (H.2), há uma correspondência biunívoca entre soluções periódicas lentamente espiralantes de $(2.1-\alpha)$ e pontos fixos não triviais de $A_{\alpha}$.

Desta forma podemos representar por

$$
\Sigma=\left\{(\alpha, \varphi) \in(0, \infty) \times K \mid \varphi \in K_{\alpha}-\{0\}, A_{\alpha}(\varphi)=\varphi\right\}
$$

o conjunto de tais funções. 
A proposição seguinte é consequência dos resultados do capítulo 2.

Proposição 4.3. Sob as hipóteses (H.1) e (H.2) valem as afirinações :

(i) $\Sigma \subset(0, \infty) \times \bar{D}_{M}$,

(ii) Para todo $\alpha>a_{0}, \quad \exists \varphi \in K \cap \bar{D}_{M}, \quad \varphi \neq 0$, tal que $(\alpha, \varphi) \in \Sigma$.

Teorema 4.2. Sejam $\Sigma$ definido em (4.3) e $\alpha_{0}$ como em (1.7). Então valem as afirmações

(i) $\exists \bar{\alpha}>0$ tal que se $(\alpha, \varphi) \in \Sigma$. então $\alpha \geq \bar{\alpha}$ e $\|\varphi\| \leq M$.

(ii) $O$ fecho $\bar{\Sigma}$ de $\Sigma$ em $(0, \infty) \times I$ é

$$
\bar{\Sigma}=\Sigma \cup\left\{\left(\alpha_{0}, 0\right)\right\}
$$

(iii) Seja $\Sigma_{0}$ a componente conexa de $\bar{\Sigma}$ contendo $\left(\alpha_{0}, 0\right)$. Então $\Sigma_{0}$ é um subconjunto ilimitado de $(0, \infty) \times K$, mais especificamente, para cada $\alpha>\alpha_{0}$, existe solução periódica lentamente espiralante $x(t ; \alpha, \varphi)$ de $(2.1-\alpha)$ tal que $(\alpha, \varphi) \in \Sigma_{0}$ e $|x(t ; \alpha, \varphi)| \leq M$ para todo $t$.

O Teorema acima consiste no resultado central deste capítulo e consiste de uma aplicação do Teorema 4.1.

Uma série de lemas a seguir nos conduzirá à prova do Teorema 4.2. O primeiro deles tem por objetivo estender o domínio da função $A$, de $\Gamma$ a todo $(0, \infty) \times \Gamma$.

Lema 4.1. Existe uma retração contínua $\rho$ de $(0, \infty) \times K$ sobre $\Gamma$. Esta retração tem a forma

$$
\rho(\alpha, \varphi)=\left(\alpha, \rho_{\alpha}(\varphi)\right)
$$

para todo $(\alpha, \varphi) \in(0, \infty) \times K$, onde $\rho_{\alpha}^{-1}(0)=\{0\}$

Demonstração: Para todos $\alpha, \beta>0$, é fácil ver que a função

$$
\begin{aligned}
h_{\alpha \beta}: K_{\alpha} & \rightarrow K_{\beta} \\
\left(h_{\alpha \beta}(\varphi)\right)(\theta) & =e^{(\alpha-\beta) \theta} \varphi(\theta),-1 \leq \theta \leq 0
\end{aligned}
$$

é um homeomorfismo com inversa $h_{\beta \alpha \alpha}$. 
Denotemos por $H_{\alpha \beta}$ o homeomorfismo de $\mathrm{K}$ em $\mathrm{K}$ dado pela extensão de $h_{\alpha \beta}$ a $\mathrm{K}$, isto é,

$$
\begin{aligned}
H_{\alpha \beta}: K & \longrightarrow K \\
\left(H_{\alpha \beta} \varphi\right)(\theta) & =e^{(\alpha-\beta) \theta} \varphi(\theta)
\end{aligned}
$$

Como $K_{0}=\left\{\varphi \in K \mid \varphi_{\jmath}(\theta)\right.$ é monótona crescente em $\left.[-1,0], \jmath=1,2\right\}$, a função

$$
\rho_{0}: K \rightarrow K_{0}
$$

definida por

$$
\left(\rho_{0} \varphi\right)(\theta)=\left(\begin{array}{c}
\max _{-1 \leq s \leq \theta} \varphi_{1}(s) \\
\max _{-1 \leq s \leq \theta} \varphi_{2}(s)
\end{array}\right)
$$

é uma retração contínua de $\mathrm{K}$ sobre $K_{0}$ e $\rho_{0}^{-1}=\{0\}$.

Definindo

$$
\begin{aligned}
& \rho_{\alpha}: K \quad \longrightarrow K_{\alpha} \\
& \rho_{\alpha}(\varphi)=h_{0 \alpha}\left(\rho_{0}\left(H_{\alpha 0}(\varphi)\right)\right)
\end{aligned}
$$

segue que $\rho_{\alpha}$ é uma retração continua de $K$ sobre $K_{\alpha}$ e $\rho_{\alpha}^{-1}(0)=\{0\}$, o que completa a prova. $\diamond$

Podemos agora definir uma extensão $A^{\star}$ de A por:

$$
\begin{array}{cl}
A^{\star}:(0, \infty) \times K & \longrightarrow K \\
A^{\star}(\alpha, \varphi) & =A(\rho(\alpha, \varphi))
\end{array}
$$

e também

$$
\begin{aligned}
A_{\alpha}^{\star}: K & \longrightarrow K \\
A_{\alpha}^{\star}(\varphi) & =A^{\star}(\alpha, \varphi) .
\end{aligned}
$$

As igualdades

$$
\begin{gathered}
\rho_{\alpha}(\varphi)=\varphi, \quad \varphi \in K_{\alpha} \\
A_{\alpha}^{\star}(\varphi)=A^{\star}(\alpha, \varphi)=A(\rho(\alpha, \varphi))=A\left(\alpha, \rho_{\alpha}(\varphi)\right)=A_{\alpha}\left(\rho_{\alpha}(\varphi)\right)
\end{gathered}
$$


implicam que o conjunto dos pontos fixos de $A_{\alpha}^{\star}$ é precisamente o mesmo que o conjunto dos pontos fixos de $A_{\alpha}$. Desse modo,

$$
\Sigma=\left\{(\alpha, \varphi) \in(0, \infty) \times K \mid A_{\alpha}^{\star}(\varphi)=\varphi \text { e } \varphi \neq 0\right\}
$$

É fácil provar que $\bar{\Sigma} \subset \Sigma \cup[(0, \infty) \times\{0\}] \cup\left[\{0\} \times \Lambda^{*}\right]$, observadas as continuidades de A e de $\rho_{\alpha}(\varphi)$ em relação a $\alpha$.

Lema 4.2..$^{\star}$ é completamente continua.

Demonstração: A aplicação $\rho$ do Lema 4.1 leva conjuntos limitados de $(0, \infty) \times K$ em conjuntos limitados de $\Gamma$.

De fato, como

$$
\left(\rho_{\alpha} \varphi\right)(\theta)=\left(\begin{array}{c}
\max _{-1 \leq s \leq \theta} e^{\alpha(s-\theta)} \varphi_{1}(s) \\
\max _{-1 \leq s \leq \theta} e^{\alpha(s-\theta)} \varphi_{2}(s)
\end{array}\right)
$$

é fácil ver que se

$$
R(l)=\left\{\varphi \in \mathcal{C} \mid-l<\varphi_{j}(\theta)<l, \theta \in[-1,0], \jmath=1,2\right\}
$$

então

$$
\rho((a, b) \times[R(l) \cap K]) \subset(a, b) \times \bigcup_{\alpha \in(a, b)} \rho_{\alpha}(R(l) \cap K) \subset(a, b) \times\left[R(l) \cap I^{*}\right] .
$$

O lema segue naturalmente deste fato, da continuidade de $\rho$ e da Proposição 4.2. $\diamond$ A seguir provaremos que $\Sigma$ não se aproxima de $\{0\} \times K$. As hipóteses (H.3) e (H.4), inseridas neste capítulo, nos dão uma condição suficiente para isso.

Lema 4.3. Consideremos a equação $(2.1-\alpha)$ e hipóteses (H.1), (H.2), (H.3) e (H.4). Então existem $\xi>0$ e $\alpha^{\prime}>0$ tais que para toda solução lentamente espiralante de $(2.1-\alpha)$ com $0<\alpha<\alpha^{\prime}$ se tem

$$
x(\bar{t}):=\max _{t \in \mathbf{R}}|x(t)|>\xi
$$


Demonstração: Suponhamos que não valha tal afirmaçãoE்ntão existem sequências $\left(\alpha_{n}\right)$, $0<\alpha_{n} \rightarrow 0, \mathrm{e}\left(x_{n}(t)\right)$ de soluçòes lentamente espiralantè de $\left(2.1-\alpha_{n}\right)$ tais que

$$
x_{n}\left(t_{n}\right):=\max _{t \in \mathbb{R}}\left|x_{n}(t)\right| \rightarrow 0
$$

quando $n \rightarrow \infty$.

Além disso $x_{n}\left(t_{n}\right) \neq 0$ para todo $\mathrm{n}$ e $\left(\left|x_{n}\left(t_{n}\right)\right|\right)$ e $\left(\alpha_{n}\right)$ são decrescentes, tomando subsequências se necessário.

As hipóteses (H.1) e (H.2) implicam na existência de uma constante $\Omega>1$ tal que

$$
|F(x)| \leq \Omega|x|, \text { se }|x| \leq M
$$

Assim,

$$
\dot{x}_{n}\left(t_{n}\right)=\alpha_{n}\left[-x_{n}\left(t_{n}\right)+F\left(x_{n}\left(t_{n}-1\right)\right)\right]=\mathcal{O}\left(\alpha_{n}\left|x_{n}\left(t_{n}\right)\right|\right)
$$

quando $n \rightarrow \infty$.

Por outro lado,

$$
\begin{gathered}
F\left(x_{n}\left(t_{n}-1\right)\right)=\left(\begin{array}{c}
\delta_{1} x_{2 n}\left(t_{n}-1\right) \\
\delta_{2} x_{1 n}\left(t_{n}-1\right)
\end{array}\right)+\mathcal{O}\left(\left|x_{n}\left(t_{n}\right)\right|^{2}\right) . \\
x_{i n}\left(t_{n}-1\right)=x_{i n}\left(t_{n}\right)-\dot{x}_{i n}\left(\tau_{n}\right)=x_{i n}\left(t_{n}\right)+\mathcal{O}\left(\alpha_{n}\left|x_{n}\left(t_{n}\right)\right|\right.
\end{gathered}
$$

quando $n \rightarrow \infty, i=1,2$.

Tomando eventualmente subsequências, (4.6) e (4.7) implicam que

$$
F\left(x_{n}\left(t_{n}-1\right)\right)=\left(\begin{array}{l}
\delta_{1} x_{2 n}\left(t_{n}\right) \\
\delta_{2} x_{1 n}\left(t_{n}\right)
\end{array}\right)+\mathcal{O}\left(\alpha_{n}\left|x_{n}\left(t_{n}\right)\right|\right)+\mathcal{O}\left(\left|x_{n}\left(t_{n}\right)\right|^{2}\right)
$$

Da equação $(2.1-\alpha)$ segue que

$$
\frac{1}{\alpha_{n}}\left[x_{n}\left(t_{n}\right) \cdot \dot{x}_{n}\left(t_{n}\right)\right]=-\left|x_{n}\left(t_{n}\right)\right|^{2}+x_{n}\left(t_{n}\right) \cdot F\left(x_{n}\left(t_{n}-1\right)\right)
$$

e então 


$$
\begin{aligned}
\frac{1}{2 \alpha_{n}} \frac{d\left|x_{n}\left(t_{n}\right)\right|^{2}}{d t} & =-\left|x_{n}\left(t_{n}\right)\right|^{2}+\left(\delta_{1}+\delta_{2}\right) \check{x}_{1 n}\left(t_{n}\right) x_{2 n}\left(t_{n}\right)+ \\
& +\mathcal{O}\left(\alpha_{n}\left|x_{n}\left(t_{n}\right)\right|^{2}\right)+\mathcal{O}\left(\left|x_{n}\left(t_{n}\right)\right|^{3}\right)
\end{aligned}
$$

Consequentemente,

$\frac{1}{2 \alpha_{n}} \frac{d\left|x_{n}\left(t_{n}\right)\right|^{2}}{d t} \leq\left(-1+\frac{\left|\delta_{1}+\delta_{2}\right|}{2}\right)\left|x_{n}\left(t_{n}\right)\right|^{2}+\mathcal{O}\left(\alpha_{n}\left|x_{n}\left(t_{n}\right)\right|^{2}\right)+\mathcal{O}\left(\left|x_{n}\left(t_{n}\right)\right|^{3}\right)$

Como $\left|\delta_{1}+\delta_{2}\right|<2$, a desigualdade (4.11) implica que

$$
\frac{d\left|x_{n}\left(t_{n}\right)\right|^{2}}{d t}<0
$$

para n suficientemente grande, o que é uma contradição.

Lema 4.4. Consideremos as equações $(2.1-\alpha)$ satisfazendo as hipóteses (H.1) a (H.4). Então $\exists \bar{\alpha}>0$ tal que $(2.1-\alpha)$ não tem solução periódica lentamente espiralante para $0<\alpha<\dot{\alpha}$.

Demonstração: Sejam $\xi>0$ conforme o Lema 4.3 e

$$
\bar{C}_{\xi, M}:=\left\{x \in \mathbf{R}^{2}|\xi \leq| x \mid \leq M\right\}
$$

Afirmamos que:

$\exists \varepsilon>0$ tal que se $x \in \bar{C}_{\xi, M}, \quad y \in \bar{B}_{M}, \quad|y-F(x)|<\varepsilon$ então $x \cdot y<|x|^{2}$.

De fato, se isto não valesse, existiriam sequências $\left(x_{n}\right) \in \bar{C}_{\xi, M},\left(y_{n}\right) \in \bar{B}_{M}$ tais que

$$
\left|y_{n}-F\left(x_{n}\right)\right|<\frac{1}{n} \text { e } x_{n} \cdot y_{n} \geq\left|x_{n}\right|^{2}
$$

Mas, nesse caso,

$$
x_{n} \rightarrow x_{0} \in \bar{C}_{\xi, M}, \quad y_{n} \rightarrow F\left(x_{0}\right) \in \bar{B}_{M}
$$

e então 


$$
x_{0} \cdot F\left(x_{0}\right) \geq\left|x_{0}\right|^{2}
$$

o que é uma contradição.

Seja

$$
0<\bar{\alpha}<\min \left\{\frac{\nu}{2 M}, \alpha^{\prime}\right\}
$$

$\operatorname{com} \nu=\nu(\varepsilon)>0$ da continuidade uniforme da funçãọ $\mathrm{F}$ restrita à $\bar{B}_{M}$ e $\alpha^{\prime}$ dado no Lema 4.3.

Se $0<\alpha<\bar{\alpha}$ e $x(t)$ é uma solução periódica lentamente espiralante de $(2.1-\alpha)$, então, pelo Lema 4.3 ,

$$
|x(\bar{t})|:=\max _{t \in \mathbf{R}}|x(t)|>\xi
$$

Além disso,

$$
|x(\bar{t})-x(\bar{t}-1)|=\alpha\left|-\int_{\bar{t}-1}^{\bar{t}} x(s) d s+\int_{\bar{t}-1}^{t} F(x(s-1)) d s\right| \leq 2 M \alpha<2 M \bar{\alpha}<\nu .
$$

Consequentemente,

$$
|F(x(\bar{t}))-F(x(\bar{t}-1))|<\varepsilon
$$

Em vista da afirmação feita no início da demonstração, isto implica que

$$
x(\bar{t}) \cdot F(x(\bar{t}-1))<|x(\bar{t})|^{2}
$$

e então

$$
\frac{1}{2 \alpha} \frac{d|x(\bar{t})|^{2}}{d t}=-|x(\bar{t})|^{2}+F(x(\bar{t}-1)) \cdot x(\bar{t})<0
$$

o que é uma contradição. $\diamond$

Em vista do Lema anterior segue que $\bar{\Sigma} \subset \Sigma \cup[(0, \infty) \times\{0\}]$ 
Lema 4.5. Sejam $0<\alpha<\alpha_{0}$ e a equação $(2.1-\alpha)$ com $F$ satisfazendo (H.1). Então $\exists \nu>0$ tal que $\forall \varepsilon>0, \exists T_{\xi} \geq 0$ tal que

$$
\sup _{i \geq T_{\boldsymbol{c}}}|x(t ; \alpha, \varphi)|<\varepsilon
$$

sempre que $\varphi \in \mathcal{C}$ satisfaça $\|\varphi\|<\nu$.

Demonstração: Conforme análise da equação característica feita no Capítulo 1 , todas as suas raizes têm parte real negativa se $0<\alpha<\alpha_{0}$.

Consequentemente, a solução nula de $(2.1-\alpha)$ é uniformemente assintóticamente estável, como segue de [9]. $\diamond$

Lema 4.6. Seja $A_{\alpha}^{\star}: K \rightarrow K$ a função definida em. (4.6). Então, 0 é ponto atrativo de $A_{\alpha}^{\star}$ se $0<\alpha<\alpha_{0}$ e é ponto ejetivo se $\alpha>\alpha_{0}$.

Demonstração: Se $0<\alpha<\alpha_{0}$, o Lema 4.5.. implica que 0 é ponto atrativo de $A_{\alpha}^{\star}$, com $U_{0}$ da definição 4.1 dada por $U_{0}=\{\varphi \in \mathcal{C} \mid\|\varphi\|<\nu\}$.

Se $\alpha>\alpha_{0}$, a ejetividade da origem para $A_{\alpha}: K_{\alpha} \rightarrow K_{\alpha}$ provada no Lema 2.14, juntamente com a observação que $A_{\alpha}^{\star}(K) \subset K_{\alpha}$ e $A_{\alpha}^{\star n}(\varphi)=A_{\alpha}^{n}\left(\rho_{\alpha}(\varphi)\right)$ para todo inteiro $n \geq 1$ garantem a afirmação. $\diamond$

Definição 4.3: Seja $J_{0}$ um subconjunto compacto de $\left(\alpha_{0}, \infty\right)$. Dizemos que 0 é um ponto fixo uniformemente ejetivo de $\left\{A_{\alpha}^{\star}, \alpha \in J_{0}\right\}$ se existir uma vizinhança aberta $W$ de 0 em $\mathrm{K}$ tal que para todo $\alpha \in J_{0}$ e toda $\varphi \in W-\{0\}$, existe inteiro positivo $n=n(\alpha, \varphi)$ para o qual $\left(A_{\alpha}^{\star}\right)^{n} \varphi \notin W$.

Observemos que, no caso de $J_{0}$ se reduzir a um único ponto, a definição acima se resume na definição clássica de ejetividade.

Vamos provar que a origem é um ponto fixo uniformemente ejetivo de $\left\{A_{\alpha}^{\star}, \alpha \in J_{0}\right\}$ através de um lema análogo ao Lema 2.14. Conforme foi dito no Capítulo 2, muitos dos resultados provados lá estavam voltados para o caso presente, mais geral.

Retomemos as considerações sobre as decomposições de $\mathcal{C}$ segundo os autovalores $\lambda=\lambda(\alpha)$ os quais satisfazem $0<\Im \lambda<\frac{\pi}{2}$, conforme fizemos no capítulo 2 , e chamemos de 
$b_{\alpha}$ o vetor-coordenada de $\varphi \in \mathcal{C}$ no subespaço $P_{\alpha}$, isto é,

$$
\pi_{\alpha} \varphi=\rho_{\alpha} b_{\alpha}
$$

Podemos, então enunciar:

Lema 4.7. Seja $J_{0}$ um subconjunto compacto de $\left(\alpha_{0}, \infty\right)$. Para cada $\alpha \in J_{0}$, existe uma forma quadrática definida positiva em $\mathcal{C}, V_{\alpha}(\varphi)=b_{\alpha}^{\top} D_{\alpha} b_{\alpha}$, com a seguinte propriedade:

$$
\forall p>0, \quad \exists \delta_{0}>0 \text { tal que } \forall \delta, \quad 0<\delta \leq \delta_{0}
$$

se $\varphi$ satisfaz $\|\varphi\| \leq \delta$ e $V_{\alpha}(\varphi) \geq p^{2} \delta^{2}$, então

$$
\dot{V}_{\alpha}^{\star}(\varphi):=\liminf _{t \rightarrow 0_{+}} \frac{1}{t}\left[V_{\alpha}\left(x_{t}(\cdot ; \alpha, \varphi)-V_{\alpha}(\varphi)\right]>\frac{1}{2 \gamma_{\alpha}} V_{\alpha}(\varphi)>0\right.
$$

para alguma constante $\gamma_{\alpha}>0$, onde $x_{t}(\cdot ; \alpha, \varphi)$ é solução de $(2.1-\alpha)$. Além disso, $\delta_{0}$ pode ser tomado independente de $\alpha$.

Omitiremos a demonstração deste Lema por tratar-se de simples repetição das provas de Chow e Hale em [5, Lema 3.1] ou [9, capítulo 10, Lema 1.1].

$A$ independência em $\alpha$ do $\delta_{0}$ justifica-se pela continuidade em relação ao parâmetro $\alpha$ das quantidades envolvidas, segundo resultados de [9, capítulo 7].

Lema 4.8. Seja $J_{0}$ um subconjunto compacto de $\left(\alpha_{0}, \infty\right)$ e suponhamos que as seguintes condições estejam satisfeitas:

(i) Para cada $\alpha \in J_{0}$, existe un autovalor $\lambda=\lambda(\alpha)$ de (2.11- $\left.\alpha\right) \operatorname{com} \Re \lambda>0$.

(ii) Para cada $\alpha \in J_{0}$, existe um subconjunto $K_{\alpha} \subset \mathcal{C}$, fechado e convexo, $0 \in K_{\alpha}$, e uma função continua

$$
\tau_{\alpha}: K_{\alpha}-\{0\} \rightarrow[\rho, \infty), \quad \rho>0
$$

tal que a função

$$
\begin{aligned}
A_{\alpha}: K_{\alpha} & \longrightarrow \mathcal{C} \\
A_{\alpha}(\varphi) & =x_{\tau_{\alpha}(\varphi)}(\cdot ; \alpha, \varphi), \quad \varphi \neq 0 \\
A_{\alpha}(0) & =0
\end{aligned}
$$

é completamente contínua e $A_{\alpha} K_{\alpha} \subset K_{\alpha}$. 
(iii) $\forall 0<a<M$,

$$
\left.\inf \left\{\left\|\pi_{\alpha} x_{t}\right\| \mid x_{t}=x_{t} \cdot ; \alpha, \varphi\right), \alpha \in J_{0}, \varphi \in K_{\alpha},\|\varphi\|=a, 0 \leq t \leq \tau_{\alpha}(\varphi)\right\}>0
$$

(iv) Dado $G \subset \mathcal{C}$ aberto, $0 \in G$, existe uma vizinhança $V$ de 0 em $\mathcal{C}$ tal que $x_{t}(\cdot ; \alpha, \varphi) \in G$ se $\alpha \in J_{0}, \varphi \in V \cap K_{\alpha}, \varphi \neq 0,0 \leq t \leq \tau_{\alpha}(\varphi)$.

Então 0 é ponto fixo uniformemente ejetivo de $\left\{A_{\alpha}^{\star}, \alpha \in J_{0}\right\}$.

Demonstração: Sejam $\alpha \in J_{0}, \varphi \in K_{\alpha}$ e $x_{t}=x_{t}(\cdot ; \alpha, \varphi)$ solução não nula de (2.1-a).

Chamando de $b_{\alpha}(t)$ a coordenada de $\frac{x_{t}}{\left\|x_{t}\right\|}$ em $P_{\alpha}$ e considerando a função $V_{\alpha}(\varphi)$ do Lema 4.7 , segue que

$$
V_{\alpha}\left(\frac{x_{t}}{\left\|x_{t}\right\|}\right)=b_{\alpha}(t)^{\top} D_{\alpha} b_{\alpha}(t)=\left|b_{\alpha}(t)\right|^{2} \frac{b_{\alpha}(t)^{\top}}{\left|b_{\dot{\alpha}}(t)\right|} D_{\alpha} \frac{b_{\alpha}(t)}{\left|b_{\alpha}(t)\right|} \geq\left|b_{\alpha}(t)\right|^{2} \beta_{\alpha}^{2}
$$

onde $\beta_{\alpha}^{2}:=\min \left\{b_{\alpha}^{\top} D_{\alpha} b_{\alpha}|| b_{\alpha} \mid=1\right\}$.

Então

$$
\left\|\pi_{\alpha} \frac{x_{t}}{\left\|x_{t}\right\|}\right\|^{2} \leq\left\|\rho_{\alpha}\right\|^{2}\left|b_{\alpha}(t)\right|^{2} \leq \frac{\left\|\rho_{\alpha}\right\|^{2}}{\beta_{\alpha}^{2}} V_{\alpha}\left(\frac{x_{t}}{\left\|x_{t}\right\|}\right) \leq \mu^{2} V_{\alpha}\left(\frac{x_{t}}{\left\|x_{t}\right\|}\right)
$$

onde

$$
\mu^{2}=\frac{\max _{\alpha \in J_{0}}\left\|\rho_{\alpha}\right\|^{2}}{\min _{\alpha \in J_{0}} \beta_{\alpha}^{2}}
$$

está bem definida, em vista da continuidade em $\alpha$ de $\rho_{\alpha}$ e $\beta_{\alpha}^{2}$.

Segue de (4.13) e da hipótese (iii) que

$$
\inf \left\{V_{\alpha}\left(\frac{x_{t}}{\left\|x_{t}\right\|}\right) \mid \alpha \in J_{0}, x_{t}=x(\cdot ; \alpha, \varphi), \varphi \in K_{\alpha},\|\varphi\|=a, 0 \leq t \leq \tau_{\alpha}(\varphi)\right\}=\Upsilon^{2}>0 .
$$

Fixemos $p, 0<p<\Upsilon$ e seja $\delta_{0}$ de acordo com o Lema 4.7, independente de $\alpha$.

Então para todo $\delta, 0<\delta \leq \delta_{0}$, se

$$
\|\varphi\| \leq \delta, V_{\alpha}(\varphi) \geq p^{2} \delta^{2} \Rightarrow \dot{V}_{\alpha}^{\star}(\varphi)>\frac{1}{2 \gamma_{\alpha}} V_{\alpha}(\varphi)>0
$$


Para cada $a \in J_{0}$, e $0<\delta \leq \delta_{0}$, definamos

$$
G_{\alpha}(\delta)=\left\{\varphi \in \mathcal{C}\|\| \varphi \|<\delta, V_{\alpha}(\varphi)<p^{2} \delta^{2}\right\}
$$

$G_{\alpha}(\delta)$ é aberto e $0 \in G_{\alpha}(\delta)$.

A continuidade em $(\alpha, \varphi)$ da função escalar $V_{\alpha}(\varphi)$ garante a existência de um aberto Oo tal que

$$
O_{\delta} \subset G_{\alpha}(\delta)
$$

para todo $\alpha \in J_{0}$.

Segue da hipótese (iv) a existência de $\eta>0$ tal que $D_{\eta} \subset O_{\delta_{0}}$ e

$$
\forall \alpha>0, \forall \psi \in D_{\eta} \cap K_{\alpha}, \psi \neq 0
$$

se tem

$$
x_{t}(\cdot ; \alpha, \psi) \in O_{\delta_{0}}
$$

para $0 \leq t \leq \tau_{a}(\psi)$

Para $\alpha \in J_{0}, \psi \in D_{\eta} \cap K_{\alpha}, \psi \neq 0$, sejam $t \in\left[0, \tau_{\alpha}(\psi)\right]$ fixado arbitrariamente tal que $\varepsilon=\left\|x_{t}(\cdot ; \alpha, \psi)\right\|<\delta_{0}$.

Então,

$$
V_{\alpha}\left(x_{t}\right)=\left\|x_{t}\right\|^{2} V_{\alpha}\left(\frac{x_{t}}{\left\|x_{t}\right\|}\right) \geq \varepsilon^{2} \Upsilon^{2} \geq p^{2} \varepsilon^{2}
$$

e o lema 4.7 implica que

$$
\dot{V}_{\alpha}^{\star}\left(x_{t}\right)>\frac{1}{2 \gamma_{\alpha}} V_{\alpha}\left(x_{t}\right) \geq \frac{1}{2 \gamma_{\alpha}} p^{2} \varepsilon^{2}>0
$$

isto é, para cada $\alpha \dot{\in} J_{0}, V_{\alpha}\left(x_{t}\right)$ é estritamente crescente em $t, 0 \leq t \leq \tau_{\alpha}(\psi)$.

Além disso, $\dot{V}_{\alpha}^{\star}\left(x_{t}\right)$ fica longe do zero em $\left[0, \tau_{\alpha}(\psi)\right]$, já que

$$
\dot{V}_{\alpha}^{\star}\left(x_{t}\right)>\frac{1}{2 \gamma_{\alpha}} V_{\alpha}\left(x_{t}\right) \geq \frac{1}{2 \gamma_{\alpha}} V_{\alpha}(\psi)
$$


Se $\left.A_{\alpha}(\psi)=x_{\tau_{\alpha}(\psi}\right)(\cdot ; \alpha, \psi)$ ainda permanecer em $D_{\eta} \cap K_{\alpha}$, nós podemos repetir o raciocínio para assegurar que

$$
\dot{V}_{\alpha}^{\star}\left(x_{t}\right)>\frac{1}{2 \gamma_{\alpha}} V_{\alpha}\left(x_{t}\right)>\frac{1}{2 \gamma_{\alpha}} V_{\alpha}\left(x_{\tau_{\alpha}(\psi)}\right)>\frac{1}{2 \gamma_{\alpha}} V_{\alpha}(\psi)>0
$$

para $\tau_{\alpha}(\psi) \leq t \leq \tau_{\alpha}\left(\psi^{\prime}\right)+\tau_{\alpha}\left(A_{\alpha}(\psi)\right)$ e assim sucessivamente.

Suponhamos por um momento que $A_{\alpha}^{n}(\psi) \in D_{\eta} \cap K_{\alpha}, n=1,2, \ldots$

Então $V_{\alpha}\left(x_{t}(\cdot ; \alpha, \psi)\right)$ é estritamente crescente em $t, V_{\alpha}(\psi)<p^{2} \delta_{0}^{2}$ e $\dot{V}_{\alpha}^{\star}\left(x_{t}(\cdot ; \alpha, \psi)\right)$ fica longe do zero para $t \geq 0$.

Sejam $t_{0}>0$ o primeiro instante $t$ para o qual $V_{\alpha}\left(x_{t_{0}}(\cdot ; \alpha, \psi)\right)=p^{2} \delta_{0}^{2}$ e $n_{0}$ o maior inteiro positivo tal que $\mathcal{f}_{\alpha}^{n_{0}} \psi=x_{T}$ para algum $T \leq t_{0}$.

Então, como $x_{t_{0}} \notin O_{\delta_{0}}$ pois $p^{2} \delta_{0}^{2}=V_{\alpha}\left(x_{t_{0}}\right)$, segue da construção de $\eta$ que

$$
A_{\alpha}^{n_{0}}(\psi) \notin D_{\eta} \cap K_{\alpha}
$$

o que é uma contradição.

Mostramos então que

$$
\alpha \in J_{0}, \psi \neq 0 \in D_{\eta} \cap K_{\alpha} \Rightarrow A_{\alpha}^{n}(\psi) \notin D_{\eta} \cap K_{\alpha}
$$

para algum inteiro positivo $n$.

Seja $l>0$ tal que o retângulo

$$
R(l)=\left\{\phi=\left(\begin{array}{l}
\phi_{1} \\
\phi_{2}
\end{array}\right) \in \mathcal{C} \mid-l<\phi_{\jmath}(\theta)<l, \jmath=1,2, \theta \in[-1,0]\right\}
$$

esteja contido em $D_{\eta}$.

É fácil ver que $\rho_{\alpha}\left(R(l) \cap K^{-}\right) \subset R(l) \cap K_{\alpha}, \forall \alpha \in J_{0 .}$.

Tomando o aberto $W$ da definição 4.3 como sendo $R(l) \cap K$; temos

$$
\left(A_{\alpha}^{\star}\right)^{n}(\varphi)=A_{\alpha}^{n}\left(\rho_{\alpha}(\varphi)\right) \notin W
$$

$\operatorname{para} \varphi \in W, \alpha \in J_{0} . \diamond$ 
Observemos que todas as hipóteses do Lema 4.8 estão satisfeitas para o nosso caso. De fato, (ii), (iii), (iv) seguem dos Lemas 2.7, 2.8 e 2.6, respectivamente, com $\rho=4$.

Lema 4.9. Para qualquer $J_{0} \subset(0, \infty), \alpha_{0} \notin J_{0}, \exists \varepsilon=\varepsilon\left(J_{0}\right)>0$ tal que $A^{\star}(\alpha, \varphi)^{\star} \neq \varphi$ para $\alpha \in J_{0}, 0<\|\varphi\| \leq \varepsilon$.

Demonstração : Para $J_{0} \subset\left(\alpha_{0}, \infty\right)$ a afirmação segue da ejetividade uniforme do ponto fixo 0 para a família $\left\{A_{\alpha}^{\star}, \alpha \in J_{0}\right\}$, provada no Lema 4.8 .

Para $J_{0} \subset\left(0, \alpha_{0}\right)$ segue como consequência do Corolário 3.3 que garante estabilidade asssintótica uniforme da solução nula de $(2.1-\alpha)$ cuja bacia de atração inclepende de $\alpha$ para $\alpha \in J_{0} . \diamond$

O lema acima e a inclusão $\bar{\Sigma} \subset \Sigma \cup[(o, \infty) \times\{0\}]$ implicam que $\bar{\Sigma} \subset \Sigma \cup\left(\alpha_{0}, 0\right)$.

\section{Demonstração do Teorema 4.2:}

Se tomarmos $Y=\mathcal{C}, S=K, J=(0, \infty)$ e $F=A^{\star}$, as hipóteses $(i)-(v)$ do Teorema 4.1 seguem respectivamente da expressão (4.4) e dos lemas 4.2,4.9,4.6 e 4.4.

Desse modo, se

$$
\Sigma=\left\{(\alpha, \varphi) \in(0, \infty) \times \Gamma \mid A_{\alpha}^{\star}(\varphi)=\varphi \quad \text { e } \quad \varphi \neq 0\right\}
$$

então $\bar{\Sigma}$ tem componente conexa $\Sigma_{0}$ ilimitada contendo $\left(\alpha_{0}, 0\right)$.

Isto implica que

$$
\bar{\Sigma}=\Sigma \cup\left\{\left(\alpha_{0}, 0\right)\right\}
$$

As demais afirmações seguem naturalmente do Teorema 4.1, lembrando que as soluções periódicas $x_{t}(. ; \alpha, \varphi)$ de $(2.1-\alpha)$ pertencem a $\bar{D}_{M} . \diamond$ 


\section{Referências}

1. R. Bellman e K. Cooke, "Differential-Difference Equations," Academic Press, New York, 1963.

2. F. E. Browder, On a generalization of the Schauder fixed point theorem, Duke Mathematical Journal 26 (1959), 291-303.

3. A further generalization of the Schauder fixed point theorem, Duke Mathematical Journal 32 (1965), 575-578.

4. S. Chow, Existence of periodic solutions of autonomous functional differential equations, J. Differential Equations 15 (1974), 350-378.

5. e J. Hale, Periodic solutions of autonomous equations J. Math. Anal. Appl. 66 (1978), 495-506.

6. R. Grafton, A periodicity theorem for autonomous functional differential equations, J. Differential Equations 6 (1969), 87-109.

7. K. Hadeler, Delay equations in biology, em "Functional Differential Equation and Approximations of Fixed Points." H. O. Peitgen e H. O. Walther (ed.). Lecture Notes in Mathematics 730, Springer Verlag, Berlin, Heidelberg (1979).

8. e J. Tomiuk, Periodic solutions of difference-differential equations, Arch. Rat. Mech. Anal. 65 (1977), 87-95.

9. J. K. Hale, "Theory of Functional Differential Equations," Springer Verlag,New York, 1977.

10. G. Jones, The existence of periodic solutions of $f^{\prime}(x)=-\alpha f(x-1)[1+f(x)]$," J. Math. Anal. Appl. 5 (1962), 435-450.

11. J. Mallet-Paret, Morse decompositions for delay-differential equations, J. Differential Equations 72 (1988), 270-315. 
12. e R. Nussbaum, Global continuation and asymptotic behavior for periodic solutions of a differential-delay equation, Ann. Mat. Pura Appl. IV, vol CXLV (1986), 33-128.

13. R. D. Nussbaum, A global bifurcation theorem with applications to functional differential equations J. Functional Analysis 19 (1975), 319-338.

14. Periodic solutions of some nonlinear autonomous functional differential equations, Ann. di Mat. Pura e Appl. 101 (1974), 263-306.

15. L. Pontryagin, $O n$ the zeros of some elementary transcedental functions, A.M.S. Tanslations (2) 1 (1955), 95-1-10.

16. P. Z. Táboas, Periodic solutions of a planar delay equation, Proc. Royal Soc. Edinburgh 116A (1990), 85-101. 